\title{
Microwave Assisted Synthesis of Novel Bis-Flavone Dimers as New Anticancer Agents
}

\author{
Andrew McGown ${ }^{\text {a }}$, Abby Ragazzon-Smith ${ }^{1 \mathrm{a}}$, John A. Hadfield ${ }^{2 \mathrm{a}}$, Herman Potgetier $^{3 \mathrm{~b}}$ and Patricia A. \\ Ragazzon ${ }^{* c}$
}

(The authors are required to provide their full names, the institutional affiliations and the location, with an asterisk in front of the name of the principal/corresponding author).

${ }^{a}$ Biomedical Research Centre, Environment and Life Sciences, University of Salford, Salford, UK; ${ }^{b}$ School of Science and the Environment, Manchester Metropolitan University, Manchester, UK; ${ }^{c}$ School of Pharmacy, Keele University, Staffordshire, UK

\begin{tabular}{l} 
A R T I C L E H I S T O R Y \\
\hline Received: \\
Revised: \\
Accepted: \\
DOI:
\end{tabular}

\begin{abstract}
In this study we describe a microwave based click chemistry method used to prepare a family of novel bis-flavone dimers. The substituted 7-hydroxy and 4'-hydroxy flavonoids were linked through a triazole ring. The compounds were easily synthesized and purified in high yields. The bis-flavonoids were tested on different cell lines including HCT116, HepG2, MCF7 and MOLT-4. Several analogues showed to have anticancer activity with IC50 values in the range of $20-60 \mu \mathrm{M}$. Flavonoids are known for their anticancer properties and this method provides the basis for new medicinal structures.
\end{abstract}

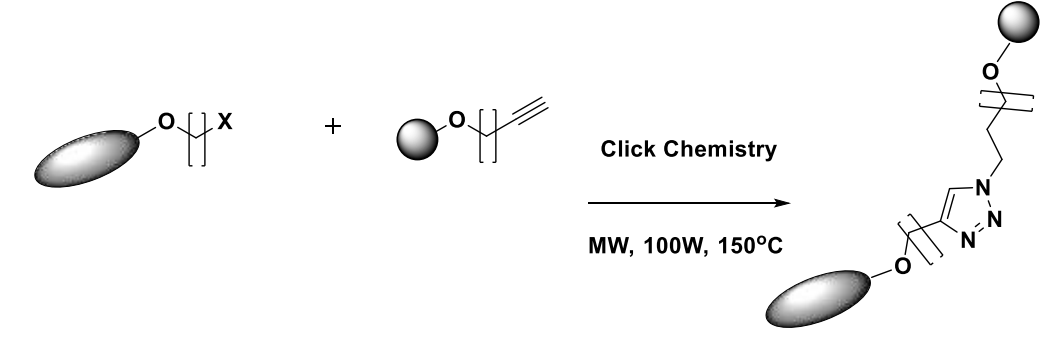

-Novel total chemical synthesis of bis-flavonoids.

-Efficient chemical ligation using Click-chemistry under microwave reaction.

-Flexible and green strategy can be used in the synthesis of a variety of analogues in the search of anticancer agents. $-60-80 \%$ Yield.

Keywords: flavonoid, bis-flavonoid, green-chemistry, click-chemistry, microwave-assisted chemistry.

\section{INTRODUCTION}

Flavonoids are a family of polyphenolic chromophores which are abundant in nature in many fruits and vegetables. The flavonoids consist of six major groups; flavones, flavanones, flavanols, flavonols, isoflavones and anthocyanins each of which have their own biological characteristics (Figure 1)[1]. Flavones, such as luteolin, have been found to exert potent anticancer, anti-inflammatory and antioxidant properties and have also been linked to the reversal of multidrug resistance in tumour cell lines[2]. Flavonoids have been found to have strong antimicrobial, anticancer, antioxidant, hepatoprotective and antiinflammatory activities[1,3]. Reports show the consumption of a diet with substantial quantities of these polyphenols produced a marked reduction in the occurrence of chronic illness such as coronary heart disease and strokes[1,3]. Traditional medicines are known to have been used to treat infections and illnesses. In many of these concoctions, high levels of phenolic compounds such as flavonoids and their derivatives can be found[4-6]. The existence of hydroxyl groups on the A-ring in the 5 and 7 positions, on the B-ring on the 2' and 6' positions (Figure 1) (1) and the double bond on the $\mathrm{C}$ ring that keeps the A- and B-rings in a planar conformation allow for intercalation of the aromatic systems with DNA[4] which are believed to be key in the inhibition of tumour growth[7,8]. Several reports have shown the presence of 2 flavonoid scaffolds linked together in different plants, such as Daemonorops draco[9], Ginkgo Biloba[10], Garcinia buehanani[11], Pentagramma triangularis[12] and Coniferae[13]; these unusual structures might provide different biological activities. 


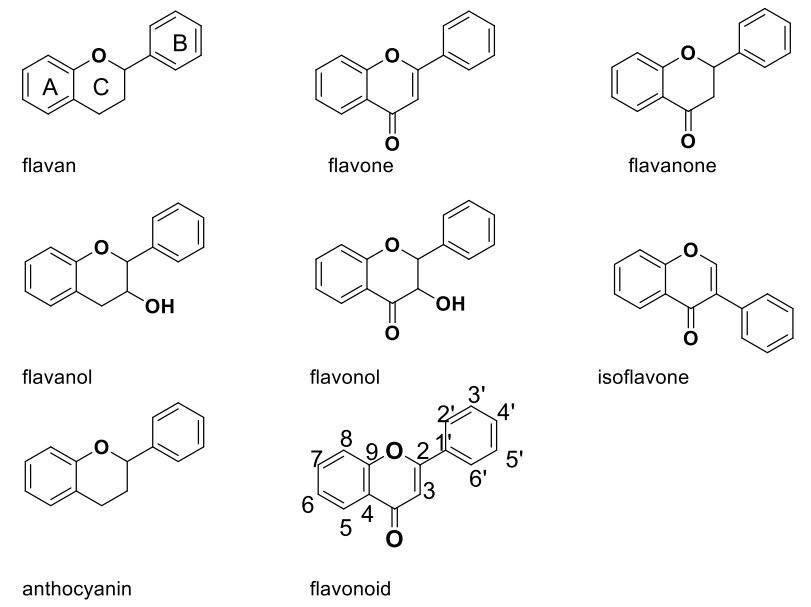

Figure 1. The structures of flavan, flavone, flavanone, flavanol, flavonol, isoflavone and anthocyanin.

In general, the synthetic chemistry of flavonoids is based on simple modifications such as hydroxyls, carbohydrates, halogens and dimerization with enzymatic reactions[14]. The versatility of a click chemistry approach allows for the exploration of novel families, using a low cost synthetic route allowing easy purification. The aim of this study was to produce a new family of symmetrically-substituted bisflavonoids using a green chemistry approach. In the first step we produced a library of mono-substituted flavones expanded from three individual core structures; 7-hydroxy-2phenyl-4H-chromen-4-one (2), 2-(3'-hydroxyphenyl)-4Hchromen-4-one (3), and 2-(4'-hydroxyphenyl)-4H-chromen4-one (4). In the second step, the substituted monomers were linked to produce bis-flavone dimers (5), which consist of two separate modified mono-flavones linked through a central triazole ring with varying overall carbon chain lengths and functionality (Figure 2).

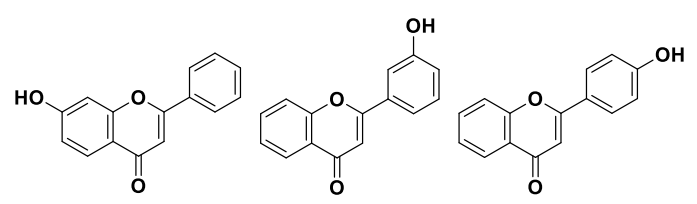

(2)

(3)

(4)

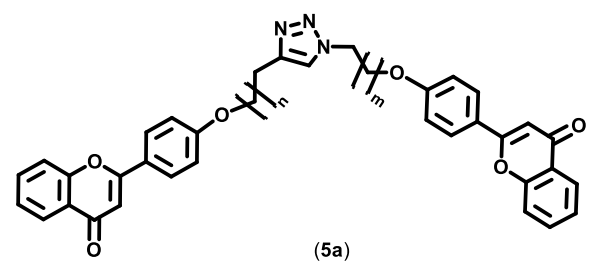

(5a)

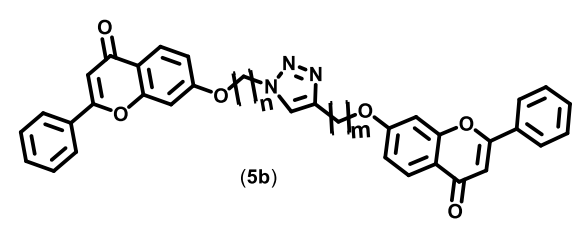

Figure 2. The structures of flavonoids prepared in this work, 7-hydroxyflavone $\quad \mathbf{2}, \quad 3$ '-hydroxyflavone $\quad \mathbf{3}, \quad 4$ '- hydroxyflavone $\mathbf{4}$ and 5a \& $\mathbf{5 b}$ examples bis-flavone dimer skeleton structure $\mathbf{5}$.

This chemistry was achieved by employing a click chemistry approach where an alkyne and an azide are reacted to form a triazole ring. Of particular note is the development of a short reaction time, high-yielding, green microwave process to synthesise the bis-flavones. These bis-flavone dimers were tested for both specificity and potency using assays of cell cycle arrest, and growth inhibition. Three active sites were identified on the mono-flavone ring structures which provided the potential for effective expansion and combination, one on the A-ring at the 7 position, and a further two on the B-ring at the 3' and 4' positions. A family of mono-flavones at position 3' was explored but they were not successful in the next stage for producing bis-flavonoids due to steric hindrance, the characterization of 2-(3'hydroxyphenyl)-4H-chromen-4-one appears is Suppl Mat.

\section{RESULTS AND DISCUSSION}

\subsection{Chemistry}

The 7-hydroxy-2-phenyl-4H-chromen-4-one (2), 2-(3'hydroxyphenyl)-4H-chromen-4-one (3), and 2-(4'hydroxyphenyl)-4H-chromen-4-one (4) flavone structures were produced using the Baker-Venkataraman method $[15,16]$. Upon the production of these core flavone structures, modification was undertaken at the corresponding hydroxyl group through the application of microwave assisted O-alkylation[17]. Other approaches were attempted to produce the mono-flavones, such as the Mitsunobu reaction, including sonication, however these were found to be much less effective providing lower yields and requiring extensive purification[18-20]. The use of a microwave reactor and less-hazardous reactants were essential in increasing the yield and improving the purification process as well as making the process more in line with a green chemistry approach. The use of a microwave reactor drastically reduced the reaction time from 12-24 hours achieving a $40-50 \%$ yield to just 0.1 hours with a yield $>85 \%$. The reaction also occurred in ethanol which has advantages over anhydrous tetrahydrofuran (THF) or anhydrous dichloromethane (DCM) as used in the Mitsunobu reaction. The respective flavone underwent expansion with a linker molecule of between 3 and 6 carbons in length with a terminal functionality of either halogen $(\mathrm{Br}$ or $\mathrm{Cl}$ ), or an alkyne group as shown in Schemes 1 and 2, to produce $6 \mathrm{e}, 6 \mathrm{f}, 7 \mathrm{~d}$ and $7 \mathrm{e}$ di-halogenated reagents were used; the reactions proceeded through a $\mathrm{S}_{\mathrm{N}} 2$ mechanism between the alkoxide on the flavonoid and the alkyl halide (or alkyl di-halide. These functional groups are essential for the following step which involved the transformation of the halide into an azide, accompanied by 1, 3-dipolar cycloaddition and the formation of the central triazole ring by in situ click chemistry[21]. The reaction was undertaken in $35 \mathrm{~mL}$ Pyrex pressure vessels, sealed with SP-D Pressure Caps both from CEM UK to which the desired flavone $\mathbf{2}, \mathbf{3}$, or $\mathbf{4}$ was added and suspended in a minimal volume of pure ethanol before the base, potassium carbonate, was added in excess. 


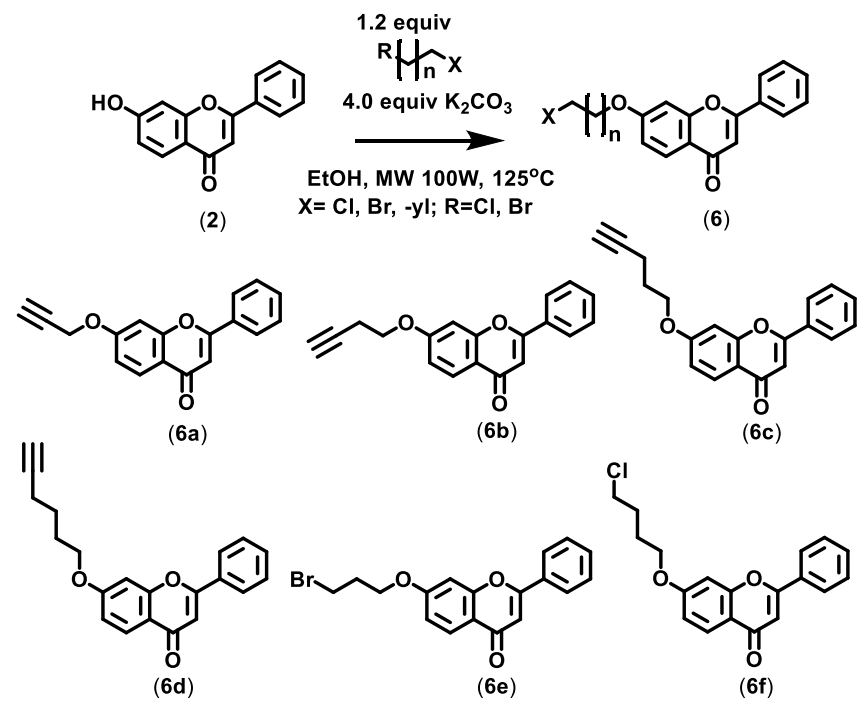

Scheme 1. Synthetic route of modified mono-flavones on the position 7 .
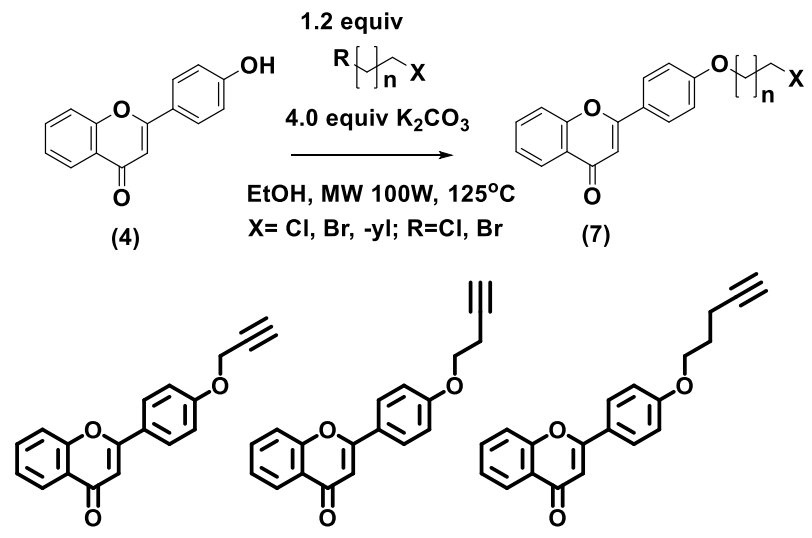

(7a)

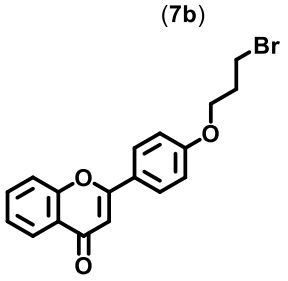

(7d)

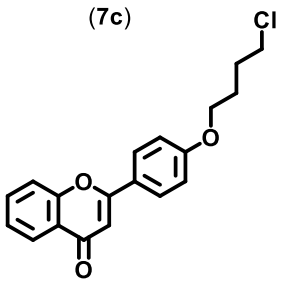

(7e)
Scheme 2. Synthetic route of modified mono-flavones of the positions 4'.

The bis-flavone dimers were produced through the application of click chemistry relying on the process of 1, 3dipolar cycloaddition whereupon an azide and alkyne functional group will form a 1,2,3-triazole ring structure[21,22] (Schemes 3 and 4). An essential step for the progression of the click reaction is the formation of the unstable azide from the halogenated mono-flavones. The initial approach involved a two-step synthetic methodology which involved the formation of flavone azide in a separate reaction before undertaking the 1,3-dipolar cycloaddition. However, it was found to be much more efficient to perform the azide formation in situ with the click chemistry[23,24]. The combination of these two steps to produce the azide in situ not only provided a one-pot, single step reaction to produce the bis-flavone dimers but also avoids the production of the highly reactive primary azide. In order to be classified as a click chemistry reaction the reaction must adhere to certain rules, such as being wide in scope, provide high yields, water and oxygen insensitivity and easy to perform with readily available reagents[25]. The formation of these dimers was performed following the click chemistry guidelines as the reaction took place in a very mild environment using a solvent system of a 1:1 ratio of water and tertiary butanol. The use of copper as a catalyst in these reactions acts to increase reaction rate and yield and is provided by both metallic $\mathrm{Cu}$ and the $\mathrm{Cu}^{2+}$ ion from $\mathrm{CuSO}_{4}$. These reagents are widely available, non-hazardous and easily removed which increases the applications of this method of synthesis. While Sharpless and colleagues reported that click chemistry could proceed at room temperature with no requirement for the input of an external energy source the formation of the bis-flavone dimers was performed under reflux[21]. This was done in order to reduce the reaction time from 48-96 hours when unheated to just 12-24 hours. A further improvement was the application of the microwave reactor as this led to a further decrease in reaction time to just 0.25 hours. The microwave reactor was ideal as an external energy source as it removed the necessity for lengthy heating by reflux further increasing this method of bis-flavone productions' green credentials. The in situ click chemistry reaction was undertaken as described in Schemes 3 and 4. The respective halogenated and azide functionalised flavones were added into a sealed microwave vial along with a slight excess of sodium azide and catalytic amounts of copper turnings, aqueous copper sulphate solution and sodium ascorbate. Sodium ascorbate acts as a reducing agent to generate the $\mathrm{Cu}(\mathrm{I})$ which is essential in the catalysis of click chemistry[18]. The materials were suspended in a mixture of water and $\mathrm{t}-\mathrm{BuOH}$ in a 1:1 ratio and heated at $150^{\circ} \mathrm{C}$ in a microwave reactor at $100 \mathrm{~W}$ power. Upon cooling the suspension was diluted with ice cold, distilled water and the contents of the vial were poured onto crushed ice leading to the precipitation of the pure bisflavone dimer which was washed, collected and dried by filtration. A total of 12 bis-flavone dimers were synthesized and characterized. The structures of the products were confirmed by ${ }^{1} \mathrm{H}$ NMR, ${ }^{13} \mathrm{CNMR}$ and IR and Mass spectral data. 


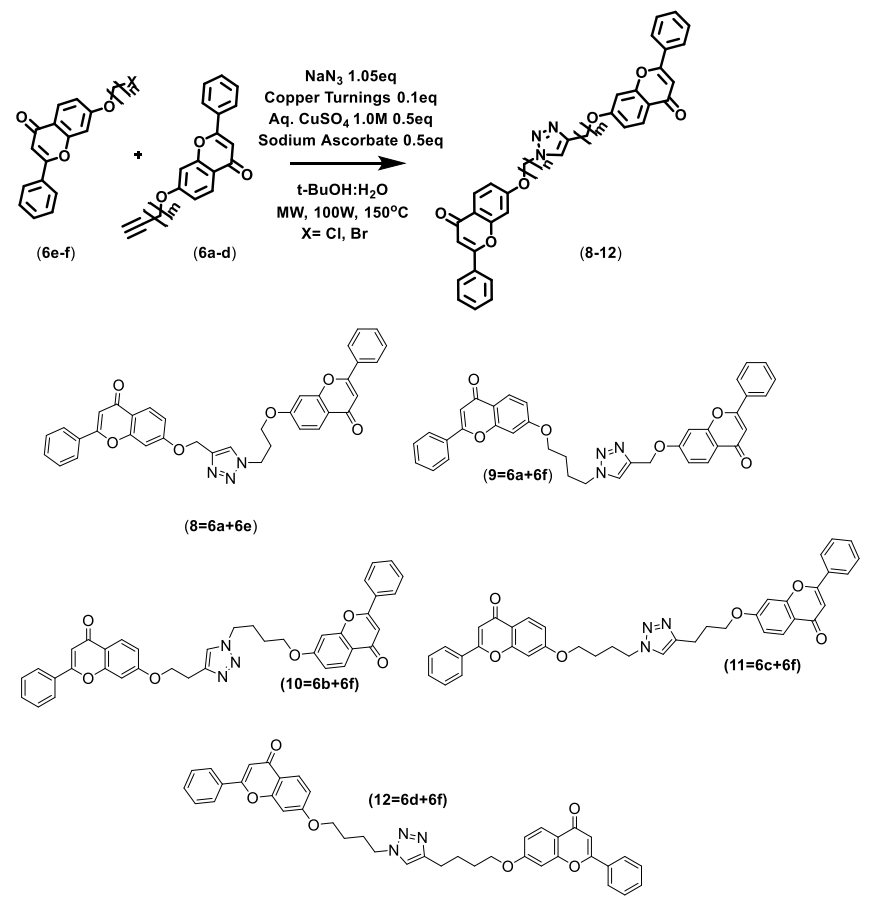

Scheme 3. Synthetic route of bis-flavone dimers from position 7.

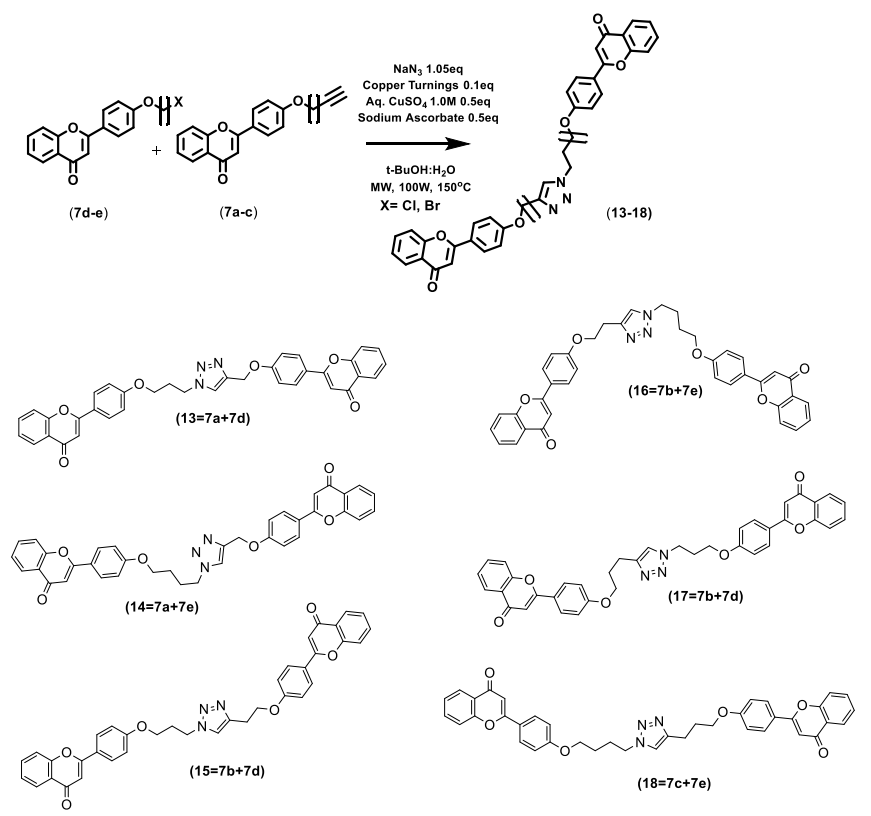

Scheme 4. Synthetic route of bis-flavone dimers from position 4'.

\subsection{Anticancer activity}

Cancer is responsible for up to 8 million deaths annually worldwide accounting for almost $13 \%$ of all global mortalities with lung, colorectal, breast and liver being some of the most commonly diagnosed types[25]. The National Cancer Institute (NCI) has identified in excess of 100 cancers each requiring specific treatment approaches[25,26]. Many factors, such as the environment (including pollutants or direct sunlight), age, weight and diet, a genetic predisposition and exposure to carcinogens and infectious agents, have the potential to increase the likelihood of cancer $[26,27]$. The growth inhibition activities of the bis-flavone dimers were determined in a series of tumour and nontumour cell lines. These cell lines represent some of the most common cancer types including leukaemia (MOLT-4), colorectal (HCT116), breast (MCF-7) and liver (Hep G2) cancers along with an immortalised keratinocyte (HaCaT) non-tumour cell line. HaCaT is used to determine if the growth inhibition activity was specific to cancerous cell lines as an ideal characteristic of an anticancer agent would be a lack of activity on non-cancerous cells (Table 1). From the growth inhibition studies several active compounds were identified. Bis-flavone dimers $8, \mathbf{1 4}, 15$ and 16 were found to produce $\mathrm{IC}_{50}$ concentrations in the low micromolar range in several cell lines. The degree of growth inhibition of each dimer was dependent on the cell line. The bis-flavone dimer 9, joined by the 7 position in an $\mathrm{L}$ shape, was found to be active on HepG2 and MOLT4 (27 and $56 \mu \mathrm{M}$ respectively). Dimers joined at the 4'-position with a more $U$ shaped structure, showed more activity in general with 14, 15 and 16 being all active on MOLT4, with $\mathbf{1 6}$ being active also of HepG2 and MCF7. Though 15 was active on MOLT4 (24 $\mu \mathrm{M})$, it was also active on $\mathrm{HaCaT}(47 \mu \mathrm{M})$, demonstrating an unwanted toxicity on a non-cancerous cell line. Other cell lines were tested, such as colorectal cancer Caco-2 and kidney cancer Caki-2, but none of the compounds showed activity on them. 
Table 1. Effect of bis-flavone dimers 8-2 on the growth of Hep G2, MCF-7, HCT116, MOLT-4 and HaCaT cell lines following 72 hours of drug treatment. Values are the concentration required to cause a $50 \%$ decrease in cell growth compared to untreated control (IC 50$)$.

\begin{tabular}{|c|c|c|c|c|c|}
\hline \multirow{2}{*}{ Dimer } & \multicolumn{5}{|c|}{$\mathrm{IC}_{50}(\mathrm{x} \pm \mathrm{SD}) \mu \mathrm{M}^{*}$} \\
\cline { 2 - 6 } & Hep G2 & MCF-7 & HCT116 & MOLT-4 & HaCaT \\
\hline $\mathbf{C P Z}$ & $2.69 \pm 0.75$ & $2.71 \pm 0.21$ & $6.32 \pm 0.76$ & $20.42 \pm 1.09$ & $12.13 \pm 0.078$ \\
\hline $\mathbf{8}$ & $27.25 \pm 0.37$ & $>100$ & $>100$ & $55.99 \pm 0.21$ & $>100$ \\
\hline $\mathbf{9}$ & $>100$ & $>100$ & $>100$ & $>100$ & $>100$ \\
\hline $\mathbf{1 0}$ & $>100$ & $>100$ & $>100$ & $>100$ & $>100$ \\
\hline $\mathbf{1 1}$ & $>100$ & $>100$ & $>100$ & $>100$ & $>100$ \\
\hline $\mathbf{1 2}$ & $>100$ & $>100$ & $>100$ & $>100$ & $>100$ \\
\hline $\mathbf{1 3}$ & $>100$ & $>100$ & $>100$ & $>100$ & $>100$ \\
\hline $\mathbf{1 4}$ & $>100$ & $>100$ & $>100$ & $68.60 \pm 0.97$ & $>100$ \\
\hline $\mathbf{1 5}$ & $>100$ & $>100$ & $>100$ & $24.3 \pm 1.36$ & $>100$ \\
\hline $\mathbf{1 6}$ & $50.05 \pm 7.75$ & $46.94 \pm 1.52$ & $>100$ & $52.64 \pm 3.53$ & $>100$ \\
\hline $\mathbf{1 7}$ & $>100$ & $>100$ & $>100$ & $>100$ & $>100$ \\
\hline $\mathbf{1 8}$ & $>100$ & $>100$ & $>100$ & $>100$ & $>100$ \\
\hline
\end{tabular}

$* \mathrm{IC}_{50}$ values are expressed as a mean value $\pm \mathrm{SD}$ from two independent experiments performed in triplicate. CPZ:

chlorpromazine, positive control

\section{EXPERIMENTAL SECTION}

\subsection{Materials and instruments}

Unless stated otherwise all chemicals and reagents were used as received. Synthetic reagents were purchased from SigmaAldrich, Thermo Fisher Scientific, Apollo Scientific or TCIUK.

${ }^{1} \mathrm{H}$ and ${ }^{13} \mathrm{C}$ NMR spectra were measured on a Bruker AC400 NMR spectrometer using dimethyl sulfoxide- $\mathrm{d}_{6}$ as the solvent and tetramethylsilane (TMS; $\delta=0$ ) as the internal reference. All solvents used for NMR analysis were purchased from Cambridge Isotope Laboratories Incorporated. High resolution mass spectra were recorded on an Agilent Technologies 1260 series HPLC coupled with an Agilent 6120b Quadrupole LC/MS with ESI source. Infrared spectra were measured on a Thermo Scientific Nicolet iS10 and melting point analysis was performed on a Stuart Melting Point SMP20. Microwave assisted reactions were performed in a CEM Discover SP microwave reactor. Silica gel chromatography was performed using silica gel $60 \AA$ with a pore size of 40- $63 \mu \mathrm{m}$ (Fluorochem Limited). Silica thin layer chromatography was performed on pre-coated aluminium sheets with a $0.2 \mathrm{~mm}$ thickness obtained from Thermo Fisher Scientific. Anhydrous tetrahydrofuran was distilled over sodium and benzophenone prior to use. All other anhydrous solvents were purchased from Thermo Fisher Scientific.

\subsection{General Procedure for the Synthesis of flavonoid skeleton.}

A general synthetic method was employed following Ares' approach[7,15] employing hydroxyacetophenone and benzoyl chloride derivatives in dry THF and under an inert atmosphere.

3.3. General Procedure for the Synthesis of modified mono-flavones using microwave assisted $\mathbf{O}$-alkylation
The general synthetic method was employed to produce the alkyl modified mono-flavones. Inside a $10 \mathrm{ml}$ Pyrex pressure vessel, flavone derivative $(2.1 \mathrm{mmol})$ and an excess of potassium carbonate $(8.4 \mathrm{mmol})$ were suspended in ethanol $(5.0 \mathrm{ml})$ and mixed thoroughly by use of a vortex for 60 seconds. To this mixture alkyl bromide or chloride (4.2 $\mathrm{mmol}$ ) were added before the reaction vessel was sealed with SP-D Pressure Caps and irradiated in the CEM Discover SP microwave reactor for 6 minutes at $100^{\circ} \mathrm{C}$ with $100 \mathrm{~W}$ constant power. The vessel was constantly cooled to maintain temperature during reaction and to cool down to room temperature after completion by the use of compressed air. The reaction mixture was then poured into ice water and stored at $4^{\circ} \mathrm{C}$ for 12 hours to allow slow precipitation to occur. The resulting precipitate was collected using filtration, washed with ice cold water $(2 \times 20 \mathrm{ml})$, followed by ice cold petroleum ether $(2 \times 20 \mathrm{ml})$ and allowed to air dry.

\subsection{General Synthesis of bis-flavone dimers}

The general synthetic method was employed to produce the azido- modified mono-flavones and followed by in situ click chemistry. Inside a $10 \mathrm{ml}$ Pyrex pressure vessel, flavone derivative $(0.64 \mathrm{mmol})$, sodium azide $(1.95 \mathrm{mmol})$ and copper turnings $(0.33 \mathrm{mmol})$ were suspended in a mixture of water and tert-butanol $(1: 1,3.0 \mathrm{ml})$ and vortexed for 30 seconds. To the suspension aqueous copper sulphate solution $(0.1 \mathrm{M}, 0.3 \mathrm{ml})$ and sodium ascorbate solution $(1.0 \mathrm{M}, 0.1$ $\mathrm{ml}$ ) were added and the vessel was sealed with SP-D Pressure Caps and irradiated using a CEM Discover SP microwave reactor for 15 minutes at $125^{\circ} \mathrm{C}$ with $300 \mathrm{~W}$ constant power. The vessel was constantly cooled to maintain temperature during reaction and to cool down to room temperature after completion by the use of compressed air. The contents were then poured into ice water and stored at $4^{\circ} \mathrm{C}$ for 12 hour facilitating the formation of a pale brown precipitate. The precipitate was collected, suspended in dichloromethane and passed through a silica plug. The organic filtrate was concentrated in vасио and the resulting solid was washed with aqueous hydrochloric acid $(0.25 \mathrm{M}$, 
$10.0 \mathrm{ml})$, ice cold water $(15.0 \mathrm{ml})$ and petroleum ether (15 $\mathrm{ml}$ ). The resulting solid was purified by column chromatography (silica $60 \AA$, ethyl acetate: petroleum ether 1:1) to yield bis-flavone.

\subsection{Characterisation}

Characterisation of compounds 2 to 7 are provided in the supplementary material.

7-(3-(4-(((4-Oxo-2-phenyl-4H-chromen-7-yl)oxy)methyl)1H-1,2,3-triazol-1-yl)propoxy)-2-phenyl-4H-chromen-4one $-\underline{\mathbf{8}}$. Yield: $0.258 \mathrm{~g}, 71.7 \%$; m.p. $261-263{ }^{\circ} \mathrm{C} ; \mathrm{Rf}=0.83$ (Silica $60 \AA, 1: 1$ ethyl acetate: petroleum ether, v/v); $\delta_{\mathrm{H}}$ $2.21\left(2 \mathrm{H}, \mathrm{m}, 1.5 \mathrm{~Hz}, 7.4 \mathrm{~Hz}, 15.2 \mathrm{~Hz}, 2^{\mathrm{II}}\right), 4.06(2 \mathrm{H}, \mathrm{t}, 1.5$ $\left.\mathrm{Hz}, 7.4 \mathrm{~Hz}, \mathbf{3}^{\mathrm{II}}\right), 4.25\left(2 \mathrm{H}, \mathrm{t}, 1.5 \mathrm{~Hz}, 7.5 \mathrm{~Hz}, \mathbf{1}^{\mathrm{II}}\right), 5.31(2 \mathrm{H}, \mathrm{t}$, $\left.1.4 \mathrm{~Hz}, 7.5 \mathrm{~Hz}, \mathbf{1}^{\mathbf{I V}}\right), 6.56\left(2 \mathrm{H}, \mathrm{s}, \mathbf{3}, \mathbf{3}^{\mathbf{V}}\right), 6.74(2 \mathrm{H}, \mathrm{m}, 1.7 \mathrm{~Hz}$, $\left.8.0 \mathrm{~Hz}, \mathbf{6}^{-} \mathbf{6}^{\mathrm{V}}\right), 6.97\left(1 \mathrm{H}, \mathrm{s}, \mathbf{1}^{\mathrm{III}}\right), 7.19(2 \mathrm{H}, \mathrm{dd}, 1.7 \mathrm{~Hz}, 7.8 \mathrm{~Hz}$, $\left.\mathbf{8}, \mathbf{8}^{\mathbf{V}}\right), 7.58\left(6 \mathrm{H}, \mathrm{m}, 1.5 \mathrm{~Hz}, 7.8 \mathrm{~Hz}, 15.8 \mathrm{~Hz}, \mathbf{3}^{\prime}, \mathbf{4}^{\prime}, \mathbf{5}^{\prime}, \mathbf{3}^{\mathbf{V I}}\right.$, $\left.\mathbf{4}^{\mathbf{V I}}, \mathbf{5}^{\mathbf{V I}}\right), 8.05\left(2 \mathrm{H}, \mathrm{d}, 7.8 \mathrm{~Hz}, \mathbf{5}^{\mathbf{5}} \mathbf{5}^{\mathbf{V}}\right), 8.29(4 \mathrm{H}, \mathrm{dd}, 1.5 \mathrm{~Hz}$, $\left.7.8 \mathrm{~Hz}, \mathbf{2}^{\mathbf{I}}, \mathbf{6}^{\mathbf{I}}, \mathbf{2}^{\mathbf{V I}}, \mathbf{6}^{\mathbf{V I}}\right) ; \delta_{\mathrm{C}} 49.4\left(\mathbf{3}^{\text {"' }}\right), 62.1\left(\mathbf{1}^{\mathbf{I V}}\right), 62.6\left(\mathbf{2}^{\mathbf{I I}}\right)$, $70.8\left(\mathbf{1}^{\mathbf{I I}}\right), 99.6\left(\mathbf{8}, \mathbf{8}^{\mathbf{V I}}\right), 107.3\left(\mathbf{3}, \mathbf{3}^{\mathbf{V I}}\right) 111.6\left(\mathbf{6}, \mathbf{6}^{\mathbf{V I}}\right), 117.2$ $\left(\mathbf{4}, \mathbf{4}^{\mathrm{VI}}\right), 124.3$ (Triazole, $\left.\mathbf{1}^{\mathrm{III}}\right), 126.2\left(\mathbf{2}^{\mathrm{I}}, \mathbf{6}^{\mathbf{I}}, \mathbf{2}^{\mathbf{V}}, \mathbf{6}^{\mathbf{V}}\right), 126.7(\mathbf{5}$, $\left.\mathbf{5}^{\mathbf{V I}}\right), 128.8\left(3^{\mathbf{I}}, \mathbf{5}^{\mathbf{I}}, \mathbf{3}^{\mathbf{V}}, \mathbf{5}^{\mathbf{V}}\right), 129.0\left(\mathbf{4}^{\mathbf{I}}, \mathbf{4}^{\mathbf{V}}\right), 131.2\left(\mathbf{1}^{\mathbf{I}}, \mathbf{1}^{\mathbf{V}}\right)$, 142.9 (Triazole, $\left.\mathbf{2}^{\mathbf{I I}}\right), 157.7\left(\mathbf{9}, \mathbf{9}^{\mathrm{VI}}\right), 162.0\left(\mathbf{7}, \mathbf{7}^{\mathrm{VI}}\right), 163.6(\mathbf{1}$, $\left.\mathbf{1}^{\mathbf{V I}}\right), 177.2\left(\mathrm{C}=\mathrm{O}, \mathbf{3}, 3^{\mathrm{VI}}\right)$; LCMS ESI $(\mathrm{MeOH}, 2500 \mathrm{~V}$, $\left.300^{\circ} \mathrm{C}\right)=\mathrm{m} / \mathrm{z}[\mathrm{M}+\mathrm{H}]^{+}=598.18$; FTIR (ATR diamond $4 \mathrm{~cm}^{-1}$ ): $1159 \mathrm{~cm}^{-1}$ (C-O-C, large ring $\mathrm{C}-\mathrm{O}$ stretch), 1051 and $1235 \mathrm{~cm}^{-1}$ (C-O, phenolic), $1596 \mathrm{~cm}^{-1} \quad(\mathrm{C}=\mathrm{C}$ aromatic), $1662 \mathrm{~cm}^{-1}$ (C=O, ketone), $1451 \mathrm{~cm}^{-1}$ and $1732 \mathrm{~cm}^{-1}$ (triazole ring), $2924 \mathrm{~cm}^{-1}$ (C-H, alkane), $2972 \mathrm{~cm}^{-1}$ (C-H, aromatic).

\section{7-((1-(4-((4-Oxo-2-phenyl-4H-chromen-7-yl)oxy)butyl)-} 1H-1,2,3-triazol-4-yl)methoxy)-2-phenyl-4H-chromen-4ne $-\underline{9}$.

Yield: $0.281 \mathrm{~g}, 74.9 \%$; m.p. $218-221{ }^{\circ} \mathrm{C}$; Rf $=0.83$ (Silica $60 \mathrm{~A}, 1: 1$ ethyl acetate: petroleum ether, $\mathrm{v} / \mathrm{v}) ; \delta_{\mathrm{H}} 1.87(2 \mathrm{H}$, $\left.\mathrm{m}, 1.5 \mathrm{~Hz}, 7.5 \mathrm{~Hz}, 15.5 \mathrm{~Hz}, 2^{\mathrm{II}}\right), 2.05(2 \mathrm{H}, \mathrm{m}, 1.5 \mathrm{~Hz}, 7.5 \mathrm{~Hz}$, $\left.15.5,3^{\mathrm{II}}\right), 4.05\left(2 \mathrm{H}, \mathrm{t} 1.5 \mathrm{~Hz}, 7.5 \mathrm{~Hz}, \mathbf{4}^{\mathrm{II}}\right) 4.21(2 \mathrm{H}, \mathrm{t}, 1.5 \mathrm{~Hz}$, $\left.7.5 \mathrm{~Hz}, \mathbf{1}^{\mathrm{II}}\right), 5.31\left(2 \mathrm{H}, \mathrm{t}, 1.5 \mathrm{~Hz}, 7.5 \mathrm{~Hz}, \mathbf{1}^{\mathrm{IV}}\right), 6.56(2 \mathrm{H}, \mathrm{s}, \mathbf{3}$, $\left.3^{\mathbf{v}}\right), 6.74\left(2 \mathrm{H}, \mathrm{m}, 1.7 \mathrm{~Hz}, 8.0 \mathrm{~Hz}, \mathbf{6}^{\mathbf{6}} \mathbf{6}^{\mathbf{v}}\right), 6.97\left(1 \mathrm{H}, \mathrm{s}, \mathbf{1}^{\mathrm{III}}\right)$, $7.19\left(2 \mathrm{H}, \mathrm{dd}, 1.7 \mathrm{~Hz}, 7.8 \mathrm{~Hz}, \mathbf{8}, \mathbf{8}^{\mathbf{v}}\right), 7.58(6 \mathrm{H}, \mathrm{m}, 1.5 \mathrm{~Hz}, 7.8$ $\left.\mathrm{Hz}, 15.8 \mathrm{~Hz}, \mathbf{3}^{\prime}, \mathbf{4}^{\prime}, \mathbf{5}^{\prime}, \mathbf{3}^{\mathrm{VI}}, \mathbf{4}^{\mathrm{VI}}, \mathbf{5}^{\mathrm{VI}}\right), 8.05(2 \mathrm{H}, \mathrm{d}, 7.8 \mathrm{~Hz}, \mathbf{5}$, $\left.\mathbf{5}^{\mathbf{V}}\right), 8.29\left(4 \mathrm{H}, \mathrm{dd}, 1.5 \mathrm{~Hz}, 7.8 \mathrm{~Hz}, \mathbf{2}^{\mathbf{I}}, \mathbf{6}^{\mathbf{I}}, \mathbf{2}^{\mathbf{V I}}, \mathbf{6}^{\mathbf{V I}}\right) ; \delta_{\mathrm{C}} 29.3$ (3"'), $49.4\left(\mathbf{4}^{\mathbf{4}}\right), 62.1\left(\mathbf{1}^{\text {IV }}\right), 69.1\left(\mathbf{1}^{\text {III }}, \mathbf{2}^{\text {II }}\right), 99.6\left(\mathbf{8}, \mathbf{8}^{\text {VI }}\right), 107.3$ $\left(\mathbf{2}, 2^{\mathrm{VI}}\right) 111.6\left(\mathbf{6}, \mathbf{6}^{\mathrm{VI}}\right), 117.2\left(\mathbf{4}, \mathbf{4}^{\mathrm{VI}}\right), 124.3$ (Triazole, $\left.\mathbf{1}^{\mathrm{III}}\right)$, $126.2\left(\mathbf{2}^{\mathbf{I}}, \mathbf{6}^{\mathbf{I}}, \mathbf{2}^{\mathbf{V}}, \mathbf{6}^{\mathbf{V}}\right), 126.7\left(\mathbf{5}, \mathbf{5}^{\mathbf{V I}}\right), 128.8\left(\mathbf{3}^{\mathbf{I}}, \mathbf{5}^{\mathbf{I}}, \mathbf{3}^{\mathbf{V}}, \mathbf{5}^{\mathbf{V}}\right)$, $129.0\left(\mathbf{4}^{\mathbf{I}}, \mathbf{4}^{\mathbf{V}}\right), 131.2\left(\mathbf{1}^{\mathbf{I}}, \mathbf{1}^{\mathbf{V}}\right), 142.9$ (Triazole, $\left.\mathbf{2}^{\mathbf{I I I}}\right), 157.7(\mathbf{9}$, $\left.\mathbf{9}^{\mathrm{VI}}\right), 162.0\left(\mathbf{7}, \mathbf{7}^{\mathrm{VI}}\right), 163.6\left(\mathbf{1}, \mathbf{1}^{\mathrm{VI}}\right), 177.2\left(\mathrm{C}=\mathrm{O}, \mathbf{3}, \mathbf{3}^{\mathrm{VI}}\right)$; LCMS ESI $\left(\mathrm{MeOH}, 2500 \mathrm{~V}, 300^{\circ} \mathrm{C}\right)=\mathrm{m} / z[\mathrm{M}+\mathrm{H}]^{+}=612.21$; FTIR (ATR diamond $\left.4 \mathrm{~cm}^{-1}\right): 1160 \mathrm{~cm}^{-1}$ (C-O-C, large ring $\mathrm{C}-\mathrm{O}$ stretch), 1052 and $1235 \mathrm{~cm}^{-1}$ (C-O, phenolic), $1595 \mathrm{~cm}^{-}$ ${ }^{1}\left(\mathrm{C}=\mathrm{C}\right.$ aromatic), $1661 \mathrm{~cm}^{-1}\left(\mathrm{C}=\mathrm{O}\right.$, ketone), $1451 \mathrm{~cm}^{-1}$ and $1731 \mathrm{~cm}^{-1}$ (triazole ring), $2923 \mathrm{~cm}^{-1}$ (C-H, alkane), $3081 \mathrm{~cm}^{-}$ ${ }^{1}$ (C-H, aromatic).

7-(2-(1-(4-((4-Oxo-2-phenyl-4H-chromen-7-yl)oxy)butyl)1H-1,2,3-triazol-4-yl)ethoxy)-2-phenyl-4H-chromen-4one $-\underline{\mathbf{1 0}}$.

Yield: 0.293 g, $75.1 \%$; m.p. $229-231{ }^{\circ} \mathrm{C}$; Rf $=0.83$ (Silica $60 \mathrm{~A}, 1: 1$ ethyl acetate: petroleum ether, $\mathrm{v} / \mathrm{v}) ; \delta_{\mathrm{H}} \quad 1.86(2 \mathrm{H}$, m, $\left.1.5 \mathrm{~Hz}, 7.5 \mathrm{~Hz}, 15.5 \mathrm{~Hz}, \mathbf{2}^{\mathrm{II}}\right), 2.00(2 \mathrm{H}, \mathrm{m}, 1.5 \mathrm{~Hz}, 7.5 \mathrm{~Hz}$, $\left.15.5,3^{\text {II }}\right), 3.15\left(2 \mathrm{H}, \mathrm{t}, 1.4 \mathrm{~Hz}, 6.1 \mathrm{~Hz}, 2^{\text {IV }}\right) 4.05(2 \mathrm{H}, \mathrm{t} 1.4 \mathrm{~Hz}$, $\left.7.3 \mathrm{~Hz}, 4^{\mathrm{II}}\right) 4.21\left(2 \mathrm{H}, \mathrm{t}, 1.5 \mathrm{~Hz}, 7.5 \mathrm{~Hz}, \mathbf{1}^{\mathrm{II}}\right), 4.30(2 \mathrm{H}, \mathrm{t}, 1.4$ $\left.\mathrm{Hz}, 6.1 \mathrm{~Hz}, \mathbf{1}^{\mathbf{I V}}\right), 6.56\left(2 \mathrm{H}, \mathrm{s}, \mathbf{3}, \mathbf{3}^{\mathbf{v}}\right), 6.74(2 \mathrm{H}, \mathrm{m}, 1.7 \mathrm{~Hz}, 8.0$ $\left.\mathrm{Hz}, \mathbf{6}, \mathbf{6}^{\mathrm{V}}\right), 6.97\left(1 \mathrm{H}, \mathrm{s}, \mathbf{1}^{\mathrm{III}}\right), 7.19(2 \mathrm{H}, \mathrm{dd}, 1.7 \mathrm{~Hz}, 7.8 \mathrm{~Hz}, \mathbf{8}$, $\left.\mathbf{8}^{\mathbf{V}}\right), 7.58\left(6 \mathrm{H}, \mathrm{m}, 1.5 \mathrm{~Hz}, 7.8 \mathrm{~Hz}, 15.8 \mathrm{~Hz}, \mathbf{3}^{\prime}, \mathbf{4}^{\prime}, \mathbf{5}^{\prime}, \mathbf{3}^{\mathbf{V I}}, \mathbf{4}^{\mathbf{V I}}\right.$, $\left.\mathbf{5}^{\mathrm{VI}}\right), 8.05\left(2 \mathrm{H}, \mathrm{d}, 7.8 \mathrm{~Hz}, \mathbf{5}, \mathbf{5}^{\mathrm{V}}\right), 8.29(4 \mathrm{H}, \mathrm{dd}, 1.5 \mathrm{~Hz}, 7.8$ $\left.\mathrm{Hz}, \mathbf{2}^{\mathbf{I}}, \mathbf{6}^{\mathbf{I}}, \mathbf{2}^{\mathbf{V I}}, \mathbf{6}^{\mathbf{V I}}\right) ; \delta_{\mathrm{C}} 26.3\left(3^{\prime \prime}\right), 30.5\left(\mathbf{2}^{\mathbf{I V}}\right), 49.4\left(\mathbf{4}^{\text {'” }}\right), 67.4$ $\left(\mathbf{1}^{\mathbf{I V}}\right), 69.1\left(\mathbf{1}^{\mathrm{II}}, \mathbf{2}^{\mathrm{II}}\right), 99.6\left(\mathbf{8}, \mathbf{8}^{\mathbf{V I}}\right), 107.3\left(\mathbf{2}, \mathbf{2}^{\mathrm{VI}}\right) 111.6(\mathbf{6}$, $\left.\mathbf{6}^{\mathrm{VI}}\right), 117.2\left(\mathbf{4}, \mathbf{4}^{\mathrm{VI}}\right), 124.3$ (Triazole, $\left.\mathbf{1}^{\mathrm{III}}\right), 126.2\left(\mathbf{2}^{\mathbf{I}}, \mathbf{6}^{\mathbf{I}}, \mathbf{2}^{\mathbf{V}}\right.$, $\left.\mathbf{6}^{\mathbf{V}}\right), 126.7\left(\mathbf{5}, \mathbf{5}^{\mathbf{V I}}\right), 128.8\left(\mathbf{3}^{\mathbf{I}}, \mathbf{5}^{\mathbf{I}}, \mathbf{3}^{\mathbf{V}}, \mathbf{5}^{\mathbf{V}}\right), 129.0\left(\mathbf{4}^{\mathbf{I}}, \mathbf{4}^{\mathbf{V}}\right), 131.2$ $\left(\mathbf{1}^{\mathbf{I}}, \mathbf{1}^{\mathbf{V}}\right), 142.9$ (Triazole, $\left.\mathbf{2}^{\mathrm{III}}\right), 157.7\left(\mathbf{9}, \mathbf{9}^{\mathrm{VI}}\right), 162.0\left(\mathbf{7}, \mathbf{7}^{\mathbf{V I}}\right)$, $163.6\left(\mathbf{1}, \mathbf{1}^{\mathrm{VI}}\right), 177.2\left(\mathrm{C}=\mathrm{O}, \mathbf{3}, \mathbf{3}^{\mathrm{VI}}\right)$; LCMS ESI $(\mathrm{MeOH}$, $\left.2500 \mathrm{~V}, 300^{\circ} \mathrm{C}\right)=m / z[\mathrm{M}+\mathrm{H}]^{+}=626.22$; FTIR (ATR diamond $4 \mathrm{~cm}^{-1}$ ): $1157 \mathrm{~cm}^{-1}$ (C-O-C, large ring C-O stretch), 1052 and $1237 \mathrm{~cm}^{-1} \quad\left(\mathrm{C}-\mathrm{O}\right.$, phenolic), $1594 \mathrm{~cm}^{-1} \quad(\mathrm{C}=\mathrm{C}$ aromatic), $1661 \mathrm{~cm}^{-1}$ (C=O, ketone), $1451 \mathrm{~cm}^{-1}$ and $1731 \mathrm{~cm}^{-1}$ (triazole ring), $2924 \mathrm{~cm}^{-1}$ (C-H, alkane), $3072 \mathrm{~cm}^{-1}$ (C-H, aromatic).

7-(3-(1-(4-((4-Oxo-2-phenyl-4H-chromen-7-yl)oxy)butyl)1H-1,2,3-triazol-4-yl)propoxy)-2-phenyl-4H-chromen-4one $-\underline{11}$.

Yield: $0.242 \mathrm{~g}, 60.5 \%$; m.p. $230-232{ }^{\circ} \mathrm{C} ; \mathrm{Rf}=0.85$ (Silica $60 \mathrm{~A}, 1: 1$ ethyl acetate: petroleum ether, $\mathrm{v} / \mathrm{v}) ; \delta_{\mathrm{H}} 1.86(2 \mathrm{H}$, m, $\left.1.5 \mathrm{~Hz}, 7.5 \mathrm{~Hz}, 15.5 \mathrm{~Hz}, 2^{\mathrm{II}}\right), 2.02(4 \mathrm{H}, \mathrm{m}, 1.5 \mathrm{~Hz}, 7.5 \mathrm{~Hz}$, $\left.15.5, \mathbf{3}^{\text {II }}, \mathbf{2}^{\text {IV }}\right), 2.65\left(2 \mathrm{H}, \mathrm{t}, 1.4 \mathrm{~Hz}, 7.0 \mathrm{~Hz}, \mathbf{3}^{\mathrm{IV}}\right) 4.05(2 \mathrm{H}, \mathrm{t}$ $\left.1.4 \mathrm{~Hz}, 7.3 \mathrm{~Hz}, 4^{\mathrm{II}}\right) 4.21\left(2 \mathrm{H}, \mathrm{t}, 1.5 \mathrm{~Hz}, 7.5 \mathrm{~Hz}, \mathbf{1}^{\mathrm{II}}\right), 4.30$ $\left(2 \mathrm{H}, \mathrm{t}, 1.4 \mathrm{~Hz}, 6.1 \mathrm{~Hz}, \mathbf{1}^{\mathrm{IV}}\right), 6.56\left(2 \mathrm{H}, \mathrm{s}, \mathbf{3}, \mathbf{3}^{\mathrm{V}}\right), 6.74(2 \mathrm{H}, \mathrm{m}$, $\left.1.7 \mathrm{~Hz}, 8.0 \mathrm{~Hz}, \mathbf{6}, \mathbf{6}^{\mathbf{V}}\right), 6.97\left(1 \mathrm{H}, \mathrm{s}, \mathbf{1}^{\mathrm{III}}\right), 7.19(2 \mathrm{H}, \mathrm{dd}, 1.7$ $\left.\mathrm{Hz}, 7.8 \mathrm{~Hz}, \mathbf{8}, \mathbf{8}^{\mathbf{v}}\right), 7.58\left(6 \mathrm{H}, \mathrm{m}, 1.5 \mathrm{~Hz}, 7.8 \mathrm{~Hz}, 15.8 \mathrm{~Hz}, \mathbf{3}^{\prime}\right.$, $\left.\mathbf{4}^{\prime}, \mathbf{5}^{\prime}, \mathbf{3}^{\mathbf{V I}}, \mathbf{4}^{\mathbf{V I}}, \mathbf{5}^{\mathbf{V I}}\right), 8.05\left(2 \mathrm{H}, \mathrm{d}, 7.8 \mathrm{~Hz}, \mathbf{5}, \mathbf{5}^{\mathbf{V}}\right), 8.29(4 \mathrm{H}, \mathrm{dd}$, $\left.1.5 \mathrm{~Hz}, 7.8 \mathrm{~Hz}, \mathbf{2}^{\mathbf{I}}, \mathbf{6}^{\mathbf{I}}, \mathbf{2}^{\mathbf{V I}}, \mathbf{6}^{\mathbf{V I}}\right) ; \delta_{\mathrm{C}} \quad 25.5\left(\mathbf{3}^{\mathbf{I V}}\right), 26.3\left(\mathbf{3}^{\mathbf{I I}}\right)$, $28.9\left(\mathbf{2}^{\text {IV }}\right), 49.4\left(\mathbf{4}^{\text {II }}\right), 69.1\left(\mathbf{1}^{\text {II }}, \mathbf{2}^{\text {II }}, \mathbf{1}^{\text {IV }}\right), 99.6\left(\mathbf{8}, 8^{\mathbf{V I}}\right), 107.3$ $\left(\mathbf{2}, \mathbf{2}^{\mathbf{V I}}\right) 111.6\left(\mathbf{6}, \mathbf{6}^{\mathrm{VI}}\right), 117.2\left(\mathbf{4}, \mathbf{4}^{\mathrm{VI}}\right), 124.3$ (Triazole, $\left.\mathbf{1}^{\mathrm{III}}\right)$, $126.2\left(\mathbf{2}^{\mathbf{I}}, \mathbf{6}^{\mathbf{I}}, \mathbf{2}^{\mathbf{V}}, \mathbf{6}^{\mathbf{V}}\right), 126.7\left(\mathbf{5}, \mathbf{5}^{\mathbf{V I}}\right), 128.8\left(\mathbf{3}^{\mathbf{I}}, \mathbf{5}^{\mathbf{I}}, \mathbf{3}^{\mathbf{V}}, \mathbf{5}^{\mathbf{V}}\right)$, $129.0\left(\mathbf{4}^{\mathbf{I}}, \mathbf{4}^{\mathbf{V}}\right), 131.2\left(\mathbf{1}^{\mathbf{I}}, \mathbf{1}^{\mathbf{V}}\right), 142.9$ (Triazole, $\left.\mathbf{2}^{\mathbf{I I I}}\right), 157.7(\mathbf{9}$, $\left.\mathbf{9}^{\mathbf{V I}}\right), 162.0\left(\mathbf{7}, \mathbf{7}^{\mathbf{V I}}\right), 163.6\left(\mathbf{1}, \mathbf{1}^{\mathbf{V I}}\right), 177.2\left(\mathrm{C}=\mathrm{O}, \mathbf{3}, \mathbf{3}^{\mathbf{V I}}\right)$; LCMS ESI $\left(\mathrm{MeOH}, 2500 \mathrm{~V}, 300^{\circ} \mathrm{C}\right)=\mathrm{m} / z[\mathrm{M}+\mathrm{H}]^{+}=640.25$; FTIR (ATR diamond $4 \mathrm{~cm}^{-1}$ ): $1160 \mathrm{~cm}^{-1}$ (C-O-C, large ring $\mathrm{C}-\mathrm{O}$ stretch), 1053 and $1239 \mathrm{~cm}^{-1}$ (C-O, phenolic), $1595 \mathrm{~cm}^{-}$ ${ }^{1}\left(\mathrm{C}=\mathrm{C}\right.$ aromatic), $1660 \mathrm{~cm}^{-1}$ ( $\mathrm{C}=\mathrm{O}$, ketone), $1451 \mathrm{~cm}^{-1}$ and $1731 \mathrm{~cm}^{-1}$ (triazole ring), $2958 \mathrm{~cm}^{-1}$ (C-H, alkane), $3074 \mathrm{~cm}^{-}$ ${ }^{1}$ (C-H, aromatic).

7-(4-(1-(4-((4-Oxo-2-phenyl-4H-chromen-7-yl)oxy)butyl)1H-1,2,3-triazol-4-yl)butoxy)-2-phenyl-4H-chromen-4one $-\underline{12}$.

Yield: 0.189 g, $46.1 \%$; m.p. $235-236^{\circ} \mathrm{C}$; Rf $=0.87$ (Silica $60 \mathrm{~A}, 1: 1$ ethyl acetate: petroleum ether, $\mathrm{v} / \mathrm{v}) ; \delta_{\mathrm{H}} 1.56(2 \mathrm{H}$, $\left.\mathrm{m}, 1.5 \mathrm{~Hz}, 7.5 \mathrm{~Hz}, \mathbf{3}^{\mathbf{I}}\right), 1.86(4 \mathrm{H}, \mathrm{m}, 1.5 \mathrm{~Hz}, 7.5 \mathrm{~Hz}, 15.5$ $\left.\mathrm{Hz}, \mathbf{2}^{\text {II }}, \mathbf{2}^{\text {IV }}\right), 2.02\left(2 \mathrm{H}, \mathrm{m}, 1.5 \mathrm{~Hz}, 7.5 \mathrm{~Hz}, 15.5, \mathbf{3}^{\mathbf{I I}}\right), 2.64$ $\left(2 \mathrm{H}, \mathrm{t}, 1.4 \mathrm{~Hz}, 7.3 \mathrm{~Hz}, 4^{\mathrm{IV}}\right), 4.04\left(2 \mathrm{H}, \mathrm{t} 1.5 \mathrm{~Hz}, 7.5 \mathrm{~Hz}, 4^{\mathrm{II}}\right)$, $4.21\left(4 \mathrm{H}, \mathrm{t}, 1.5 \mathrm{~Hz}, 7.5 \mathrm{~Hz}, \mathbf{1}^{\mathrm{II}}, \mathbf{1}^{\mathrm{IV}}\right), 6.56\left(2 \mathrm{H}, \mathrm{s}, \mathbf{3}, \mathbf{3}^{\mathrm{V}}\right), 6.74$ $\left(2 \mathrm{H}, \mathrm{m}, 1.7 \mathrm{~Hz}, 8.0 \mathrm{~Hz}, \mathbf{6}, \mathbf{6}^{\mathrm{V}}\right), 6.97\left(1 \mathrm{H}, \mathrm{s}, \mathbf{1}^{\mathrm{III}}\right), 7.19(2 \mathrm{H}$, dd, $\left.1.7 \mathrm{~Hz}, 7.8 \mathrm{~Hz}, \mathbf{8}, \mathbf{8}^{\mathbf{v}}\right), 7.58(6 \mathrm{H}, \mathrm{m}, 1.5 \mathrm{~Hz}, 7.8 \mathrm{~Hz}, 15.8$ $\left.\mathrm{Hz}, \mathbf{3}^{\prime}, \mathbf{4}^{\prime}, \mathbf{5}^{\prime}, \mathbf{3}^{\mathrm{VI}}, \mathbf{4}^{\mathbf{V I}}, \mathbf{5}^{\mathrm{VI}}\right), 8.05\left(2 \mathrm{H}, \mathrm{d}, 7.8 \mathrm{~Hz}, \mathbf{5}^{\mathbf{2}} \mathbf{5}^{\mathrm{V}}\right), 8.29$ $\left(4 \mathrm{H}, \mathrm{dd}, 1.5 \mathrm{~Hz}, 7.8 \mathrm{~Hz}, \mathbf{2}^{\mathbf{I}}, \mathbf{6}^{\mathbf{I}}, \mathbf{2}^{\mathbf{V I}}, \mathbf{6}^{\mathbf{V I}}\right) ; \delta_{\mathrm{C}} 25.5\left(\mathbf{4}^{\mathbf{I V}}\right), 26.0$ $\left(\mathbf{3}^{\mathbf{I V}}\right), 26.3\left(3^{\mathbf{I I}}\right), 29.2\left(2^{\mathbf{I V}}\right), 49.4\left(4^{\mathbf{I I}}\right), 68.2\left(1^{\mathbf{I V}}\right), 69.1\left(\mathbf{1}^{\mathrm{II}}\right.$, $\left.\mathbf{2}^{\mathrm{II}}\right), 99.6\left(\mathbf{8}, \mathbf{8}^{\mathrm{VI}}\right), 107.3\left(\mathbf{2}, 2^{\mathrm{VI}}\right) 111.6\left(\mathbf{6}, \mathbf{6}^{\mathrm{VI}}\right), 117.2\left(\mathbf{4}, \mathbf{4}^{\mathrm{VI}}\right)$, 124.3 (Triazole, $\left.\mathbf{1}^{\mathrm{III}}\right), 126.2\left(\mathbf{2}^{\mathbf{I}}, \mathbf{6}^{\mathbf{I}}, \mathbf{2}^{\mathbf{V}}, \mathbf{6}^{\mathbf{V}}\right), 126.7\left(\mathbf{5}, \mathbf{5}^{\mathbf{V I}}\right)$, $128.8\left(\mathbf{3}^{\mathbf{I}}, \mathbf{5}^{\mathbf{I}}, \mathbf{3}^{\mathbf{V}}, \mathbf{5}^{\mathbf{V}}\right), 129.0\left(\mathbf{4}^{\mathbf{I}}, \mathbf{4}^{\mathbf{V}}\right), 131.2\left(\mathbf{1}^{\mathbf{I}}, \mathbf{1}^{\mathbf{V}}\right), 142.9$ 
(Triazole, $\left.\mathbf{2}^{\mathrm{III}}\right), 157.7\left(\mathbf{9}, \mathbf{9}^{\mathrm{VI}}\right), 162.0\left(\mathbf{7}, \mathbf{7}^{\mathrm{VI}}\right), 163.6\left(\mathbf{1}, \mathbf{1}^{\mathbf{V I}}\right)$, $177.2\left(\mathrm{C}=\mathrm{O}, \mathbf{3}, \mathbf{3}^{\mathrm{VI}}\right)$; LCMS ESI $\left(\mathrm{MeOH}, 2500 \mathrm{~V}, 300^{\circ} \mathrm{C}\right)=$ $\mathrm{m} / \mathrm{z}[\mathrm{M}+\mathrm{H}]^{+}=654.27$; FTIR (ATR diamond $4 \mathrm{~cm}^{-1}$ ): 1161 $\mathrm{cm}^{-1}$ (C-O-C, large ring C-O stretch), 1053 and $1236 \mathrm{~cm}^{-1}$ (C-O, phenolic), $1595 \mathrm{~cm}^{-1} \quad\left(\mathrm{C}=\mathrm{C}\right.$ aromatic), $1662 \mathrm{~cm}^{-1}$ ( $\mathrm{C}=\mathrm{O}$, ketone), $1451 \mathrm{~cm}^{-1}$ and $1731 \mathrm{~cm}^{-1}$ (triazole ring), 2957 $\mathrm{cm}^{-1}$ (C-H, alkane), $3068 \mathrm{~cm}^{-1}$ (C-H, aromatic).

\section{2-(4'-(3-(4-((4-)(4-Oxo-4H-chromen-2-}

yl)phenoxy)methyl)-1H-1,2,3-triazol-1-

\section{yl)propoxy)phenyl)-4H-chromen-4-one - 13 .}

Yield: 0.192 g, $53.3 \%$; m.p. $281-283{ }^{\circ} \mathrm{C} ; \overline{\mathrm{Rf}}=0.83$ (Silica $60 \mathrm{~A}, 1: 1$ ethyl acetate: petroleum ether, $\mathrm{v} / \mathrm{v}) ; \delta_{\mathrm{H}} 2.21(2 \mathrm{H}$, m, $\left.1.3 \mathrm{~Hz}, 7.2 \mathrm{~Hz}, 14.8 \mathrm{~Hz}, 2^{\mathbf{I I}}\right), 4.06(2 \mathrm{H}, \mathrm{t}, 1.3 \mathrm{~Hz}, 7.2 \mathrm{~Hz}$, $\left.\mathbf{3}^{\mathrm{II}}\right), 4.27\left(2 \mathrm{H}, \mathrm{t}, 1.3 \mathrm{~Hz}, 7.2 \mathrm{~Hz}, \mathbf{1}^{\mathrm{II}}\right), 5.35(2 \mathrm{H}, \mathrm{t}, 1.3 \mathrm{~Hz}, 7.4$ $\left.\mathrm{Hz}, \mathbf{1}^{\mathbf{I V}}\right), 6.45\left(2 \mathrm{H}, \mathrm{s}, \mathbf{3}, \mathbf{3}^{\mathbf{V I}}\right), 6.95(4 \mathrm{H}, \mathrm{dd}, 1.4 \mathrm{~Hz}, 8.1 \mathrm{~Hz}$, $\left.\mathbf{3}^{\mathbf{I}}, \mathbf{5}^{\mathbf{I}}, \mathbf{3}^{\mathbf{V}}, \mathbf{5}^{\mathbf{V}}\right), 7.01\left(1 \mathrm{H}, \mathrm{s}, 1^{\mathrm{III}}\right), 7.17(2 \mathrm{H}, \mathrm{dd}, 1.3 \mathrm{~Hz}, 8.1 \mathrm{~Hz}$, $\left.\mathbf{8}, \mathbf{8}^{\mathbf{V I}}\right), 7.36\left(2 \mathrm{H}, \mathrm{m}, 1.3 \mathrm{~Hz}, 8.1 \mathrm{~Hz}, 15.6 \mathrm{~Hz}, \mathbf{6}, \mathbf{6}^{\mathbf{V I}}\right), 7.55$ $\left(4 \mathrm{H}, \mathrm{d}, 8.1 \mathrm{~Hz}, \mathbf{2}^{\mathbf{I}}, \mathbf{6}^{\mathbf{I}}, \mathbf{2}^{\mathbf{V}}, \mathbf{6}^{\mathbf{V}}\right), 7.70(2 \mathrm{H}, \mathrm{m}, 1.3 \mathrm{~Hz}, 8.1 \mathrm{~Hz}$, $\left.15.6 \mathrm{~Hz}, \mathbf{7}, \mathbf{7}^{\mathbf{V I}}\right), 7.85\left(2 \mathrm{H}, \mathrm{dd}, 1.3 \mathrm{~Hz}, 8.1 \mathrm{~Hz}, \mathbf{5}, \mathbf{5}^{\mathbf{V I}}\right) ; \delta_{\mathrm{C}}$ $49.4\left(\mathbf{3}^{\mathrm{II}}\right), 62.1\left(\mathbf{1}^{\mathbf{I V}}\right), 62.6\left(\mathbf{2}^{\mathrm{II}}\right), 70.8\left(\mathbf{1}^{\mathrm{II}}\right), 107.3\left(\mathbf{2}, \mathbf{2}^{\mathbf{V I}}\right)$, $115.4\left(\mathbf{3}^{\mathbf{I}}, \mathbf{5}^{\mathbf{I}}, \mathbf{3}^{\mathbf{V}}, \mathbf{5}^{\mathbf{V}}\right), 118.1\left(\mathbf{8}, \mathbf{8}^{\mathbf{V I}}\right), 122.4\left(\mathbf{1}^{\mathbf{I}}, \mathbf{1}^{\mathbf{V}}\right), 123.4(\mathbf{6}$, $\left.\mathbf{6}^{\mathbf{V I}}\right), 124.4\left(\mathbf{1}^{\mathrm{III}}\right), 125.7\left(\mathbf{5}, \mathbf{5}^{\mathrm{VI}}\right), 128.5\left(\mathbf{2}^{\mathbf{I}}, \mathbf{6}^{\mathbf{I}}, \mathbf{2}^{\mathbf{V}}, \mathbf{6}^{\mathbf{V}}\right), 131.2$ $\left(\mathbf{7}, 7^{\mathrm{VI}}\right), 142.9\left(\mathbf{2}^{\mathrm{III}}\right), 155.7\left(\mathbf{9}, \mathbf{9}^{\mathrm{VI}}\right), 158.5\left(\mathbf{4}^{\mathrm{V}}\right), 160.5\left(\mathbf{4}^{\prime}\right)$, $163.6\left(\mathbf{1}, \mathbf{1}^{\mathrm{VI}}\right), 177.2\left(\mathbf{4}, \mathbf{4}^{\mathrm{VI}}\right)$; LCMS ESI $(\mathrm{MeOH}, 2500 \mathrm{~V}$, $\left.300^{\circ} \mathrm{C}\right)=\mathrm{m} / \mathrm{z}[\mathrm{M}+\mathrm{H}]^{+}=598.19$; FTIR (ATR diamond $\left.4 \mathrm{~cm}^{-1}\right): 1161 \mathrm{~cm}^{-1}$ (C-O-C, large ring C-O stretch), 1051 and $1237 \mathrm{~cm}^{-1}$ (C-O, phenolic), $1593 \mathrm{~cm}^{-1}(\mathrm{C}=\mathrm{C}$ aromatic), 1660 $\mathrm{cm}^{-1}\left(\mathrm{C}=\mathrm{O}\right.$, ketone), $1451 \mathrm{~cm}^{-1}$ and $1731 \mathrm{~cm}^{-1}$ (triazole ring), $2980 \mathrm{~cm}^{-1}$ (C-H, alkane), $3089 \mathrm{~cm}^{-1}$ (C-H, aromatic).

\section{2-(4'-((1-(4-(4-(4-Oxo-4H-chromen-2-yl)phenoxy)butyl)-} 1H-1,2,3-triazol-4-yl)methoxy)phenyl)-4H-chromen-4one -14 .

Yield: 0.238 g, $62.6 \%$; m.p. $286-287^{\circ} \mathrm{C}$; Rf $=0.84$ (Silica $60 \mathrm{~A}, 1: 1$ ethyl acetate: petroleum ether, $\mathrm{v} / \mathrm{v}) ; \delta_{\mathrm{H}} 1.86(2 \mathrm{H}$, m, $\left.1.3 \mathrm{~Hz}, 6.8 \mathrm{~Hz}, 14.2 \mathrm{~Hz}, 2^{\mathrm{II}}\right), 2.05(2 \mathrm{H}, \mathrm{m}, 1.3 \mathrm{~Hz}, 6.8 \mathrm{~Hz}$, $3^{\text {II })} 4.05\left(2 \mathrm{H}, \mathrm{t}, 1.3 \mathrm{~Hz}, 6.8 \mathrm{~Hz}, 4^{\mathrm{II}}\right), 4.24(2 \mathrm{H}, \mathrm{t}, 1.3 \mathrm{~Hz}, 7.5$ $\left.\mathrm{Hz}, \mathbf{1}^{\mathbf{I I}}\right), 5.35\left(2 \mathrm{H}, \mathrm{t}, 1.3 \mathrm{~Hz}, 7.4 \mathrm{~Hz}, \mathbf{1}^{\mathbf{I V}}\right), 6.45\left(2 \mathrm{H}, \mathrm{s}, \mathbf{3}, \mathbf{3}^{\mathbf{V I}}\right)$, $6.95\left(4 \mathrm{H}, \mathrm{dd}, 1.4 \mathrm{~Hz}, 8.1 \mathrm{~Hz}, \mathbf{3}^{\mathbf{I}}, \mathbf{5}^{\mathbf{I}}, \mathbf{3}^{\mathbf{V}}, \mathbf{5}^{\mathbf{V}}\right), 7.01\left(1 \mathrm{H}, \mathrm{s}, 1^{\mathrm{III}}\right)$, $7.17\left(2 \mathrm{H}, \mathrm{dd}, 1.3 \mathrm{~Hz}, 8.1 \mathrm{~Hz}, \mathbf{8}, \mathbf{8}^{\mathbf{V I}}\right), 7.36(2 \mathrm{H}, \mathrm{m}, 1.3 \mathrm{~Hz}$, $\left.8.1 \mathrm{~Hz}, 15.6 \mathrm{~Hz}, \mathbf{6}, \mathbf{6}^{\mathbf{V I}}\right), 7.55\left(4 \mathrm{H}, \mathrm{d}, 8.1 \mathrm{~Hz}, \mathbf{2}^{\mathbf{I}}, \mathbf{6}^{\mathbf{I}}, \mathbf{2}^{\mathbf{V}}, \mathbf{6}^{\mathbf{V}}\right)$, $7.70\left(2 \mathrm{H}, \mathrm{m}, 1.3 \mathrm{~Hz}, 8.1 \mathrm{~Hz}, 15.6 \mathrm{~Hz}, 7,7^{\mathrm{VI}}\right), 7.85(2 \mathrm{H}, \mathrm{dd}$, $\left.1.3 \mathrm{~Hz}, 8.1 \mathrm{~Hz}, \mathbf{5}, \mathbf{5}^{\mathrm{VI}}\right) ; \delta_{\mathrm{C}} 26.3\left(\mathbf{3}^{\mathrm{II}}\right), 49.4\left(\mathbf{4}^{\mathrm{II}}\right), 62.1\left(\mathbf{1}^{\mathbf{I V}}\right)$, $69.1\left(\mathbf{1}^{\mathrm{II}}, \mathbf{2}^{\mathrm{II}}\right), 107.3\left(\mathbf{2}, \mathbf{2}^{\mathrm{VI}}\right), 115.4\left(\mathbf{3}^{\mathbf{I}}, \mathbf{5}^{\mathrm{I}}, \mathbf{3}^{\mathbf{V}}, \mathbf{5}^{\mathbf{V}}\right), 118.1(\mathbf{8}$, $\left.\mathbf{8}^{\mathrm{VI}}\right), 122.4\left(\mathbf{1}^{\mathbf{I}}, \mathbf{1}^{\mathbf{V}}\right), 123.4\left(\mathbf{6}, \mathbf{6}^{\mathbf{V I}}\right), 124.4\left(\mathbf{1}^{\mathrm{III}}\right), 125.7\left(\mathbf{5}, \mathbf{5}^{\mathbf{V I}}\right)$, $128.5\left(\mathbf{2}^{\mathbf{I}}, \mathbf{6}^{\mathbf{I}}, \mathbf{2}^{\mathbf{V}}, \mathbf{6}^{\mathbf{V}}\right), 131.2\left(\mathbf{7}, \mathbf{7}^{\mathbf{V I}}\right), 142.9\left(\mathbf{2}^{\mathbf{I I I}}\right), 155.7(\mathbf{9}$, $\left.\mathbf{9}^{\mathrm{VI}}\right), 158.5\left(\mathbf{4}^{\mathrm{V}}\right), 160.5\left(\mathbf{4}^{\prime}\right), 163.6\left(\mathbf{1}, \mathbf{1}^{\mathrm{VI}}\right), 177.2\left(\mathbf{4}, \mathbf{4}^{\mathrm{VI}}\right)$; LCMS ESI $\left(\mathrm{MeOH}, 2500 \mathrm{~V}, 300^{\circ} \mathrm{C}\right)=\mathrm{m} / z[\mathrm{M}+\mathrm{H}]^{+}=612.20$; FTIR (ATR diamond $4 \mathrm{~cm}^{-1}$ ): $1160 \mathrm{~cm}^{-1}$ (C-O-C, large ring C-O stretch), 1051 and $1237 \mathrm{~cm}^{-1}$ (C-O, phenolic), $1595 \mathrm{~cm}^{-}$ $1 \quad(\mathrm{C}=\mathrm{C}$ aromatic $), 1661 . \mathrm{cm}^{-1} \quad(\mathrm{C}=\mathrm{O}$, ketone $), 1451 \mathrm{~cm}^{-1}$ and $1731 \mathrm{~cm}^{-1}$ (triazole ring), $2974 \mathrm{~cm}^{-1}$ (C-H, alkane), $3292 \mathrm{~cm}^{-1}$ (C-H, aromatic).

2-(4'-(3-(4-(2-(4-(4-Oxo-4H-chromen-2yl)phenoxy)ethyl)-1H-1,2,3-triazol-1-yl)propoxy)phenyl)4H-chromen-4-one - $\underline{\mathbf{1 5}}$.

Yield: 0.214 g, $57.8 \%$; m.p. $278{ }^{\circ} \mathrm{C}$; Rf $=0.84$ (Silica 60A, 1:1 ethyl acetate: petroleum ether, $\mathrm{v} / \mathrm{v}) ; \delta_{\mathrm{H}} 2.21(2 \mathrm{H}, \mathrm{m}, 1.5$ $\left.\mathrm{Hz}, 7.4 \mathrm{~Hz}, 14.9 \mathrm{~Hz}, 2^{\mathbf{I I}}\right), 3.16\left(2 \mathrm{H}, \mathrm{t}, 1.5 \mathrm{~Hz}, 6.1 \mathrm{~Hz}, 2^{\mathbf{I V}}\right)$ $4.06\left(2 \mathrm{H}, \mathrm{t}, 1.5 \mathrm{~Hz}, 7.4 \mathrm{~Hz}, 3^{\mathrm{II}}\right), 4.27(4 \mathrm{H}, \mathrm{m}, 1.5 \mathrm{~Hz}, 7.4 \mathrm{~Hz}$, $\left.14.9 \mathrm{~Hz}, \mathbf{1}^{\mathrm{II}}, \mathbf{1}^{\mathbf{I V}}\right), 6.45\left(2 \mathrm{H}, \mathrm{s}, \mathbf{3}, \mathbf{3}^{\mathrm{VI}}\right), 6.95(4 \mathrm{H}, \mathrm{dd}, 1.4 \mathrm{~Hz}$, $\left.8.1 \mathrm{~Hz}, \mathbf{3}^{\mathbf{I}}, \mathbf{5}^{\mathbf{I}}, \mathbf{3}^{\mathbf{V}}, \mathbf{5}^{\mathbf{V}}\right), 7.01\left(1 \mathrm{H}, \mathrm{s}, 1^{\mathrm{III}}\right), 7.17(2 \mathrm{H}, \mathrm{dd}, 1.3 \mathrm{~Hz}$, $\left.8.1 \mathrm{~Hz}, \mathbf{8}, \mathbf{8}^{\mathbf{V I}}\right), 7.36\left(2 \mathrm{H}, \mathrm{m}, 1.3 \mathrm{~Hz}, 8.1 \mathrm{~Hz}, 15.6 \mathrm{~Hz}, \mathbf{6}^{\mathbf{2}} \mathbf{6}^{\mathbf{V I}}\right)$, $7.55\left(4 \mathrm{H}, \mathrm{d}, 8.1 \mathrm{~Hz}, \mathbf{2}^{\mathbf{I}}, \mathbf{6}^{\mathbf{I}}, \mathbf{2}^{\mathbf{V}}, \mathbf{6}^{\mathbf{V}}\right), 7.70(2 \mathrm{H}, \mathrm{m}, 1.3 \mathrm{~Hz}, 8.1$ $\left.\mathrm{Hz}, 15.6 \mathrm{~Hz}, \mathbf{7}, 7^{\mathrm{VI}}\right), 7.85\left(2 \mathrm{H}, \mathrm{dd}, 1.3 \mathrm{~Hz}, 8.1 \mathrm{~Hz}, \mathbf{5}, \mathbf{5}^{\mathbf{V I}}\right) ; \delta_{\mathrm{C}}$ $\delta \quad 30.5\left(\mathbf{2}^{\mathbf{I V}}\right), 49.4\left(\mathbf{3}^{\mathrm{II}}\right), 62.6\left(\mathbf{2}^{\mathrm{II}}\right), 67.4\left(\mathbf{1}^{\mathbf{I V}}\right), 70.8\left(\mathbf{1}^{\mathrm{II}}\right)$, $107.3\left(\mathbf{2}, \mathbf{2}^{\mathbf{V I}}\right), 115.4\left(\mathbf{3}^{\mathbf{I}}, \mathbf{5}^{\mathbf{I}}, \mathbf{3}^{\mathbf{V}}, \mathbf{5}^{\mathbf{V}}\right), 118.1\left(\mathbf{8}, \mathbf{8}^{\mathbf{V I}}\right), 122.4\left(\mathbf{1}^{\mathbf{I}}\right.$, $\left.\mathbf{1}^{\mathbf{V}}\right), 123.4\left(\mathbf{6}, \mathbf{6}^{\mathrm{VI}}\right), 124.4\left(\mathbf{1}^{\mathrm{III}}\right), 125.7\left(\mathbf{5}, \mathbf{5}^{\mathrm{VI}}\right), 128.5\left(\mathbf{2}^{\mathbf{I}}, \mathbf{6}^{\mathbf{I}}\right.$, $\left.\mathbf{2}^{\mathrm{V}}, \mathbf{6}^{\mathrm{V}}\right), 131.2\left(\mathbf{7}, \mathbf{7}^{\mathrm{VI}}\right), 142.9\left(\mathbf{2}^{\mathrm{III}}\right), 155.7\left(\mathbf{9}^{\mathbf{9}}, \mathbf{9}^{\mathrm{VI}}\right), 158.5\left(\mathbf{4}^{\mathrm{V}}\right)$, $160.5\left(\mathbf{4}^{\prime}\right), 163.6\left(\mathbf{1}, \mathbf{1}^{\mathbf{V I}}\right), 177.2\left(\mathbf{4}, \mathbf{4}^{\mathrm{VI}}\right)$; LCMS ESI $(\mathrm{MeOH}$, $2500 \mathrm{~V}, 300^{\circ} \mathrm{C}$ ) $=m / z[\mathrm{M}+\mathrm{H}]^{+}=612.20$; FTIR (ATR diamond $4 \mathrm{~cm}^{-1}$ ): $1160 \mathrm{~cm}^{-1}$ (C-O-C, large ring C-O stretch), 1051 and $1237 \mathrm{~cm}^{-1} \quad$ (C-O, phenolic), $1595 \mathrm{~cm}^{-1} \quad(\mathrm{C}=\mathrm{C}$ aromatic), $1661 \mathrm{~cm}^{-1}$ (C=O, ketone), $1451 \mathrm{~cm}^{-1}$ and $1731 \mathrm{~cm}^{-1}$ (triazole ring), $2974 \mathrm{~cm}^{-1}$ (C-H, alkane), $3292 \mathrm{~cm}^{-1}$ (C-H, aromatic).

\section{2-(4'-(2-(1-(4-(4-(4-Oxo-4H-chromen-2-}

yl)phenoxy)butyl)-1H-1,2,3-triazol-4-yl)ethoxy)phenyl)4H-chromen-4-one - $\underline{\mathbf{1 6}}$.

Yield: 0.251 g, $64.4 \%$; m.p. $285-288{ }^{\circ} \mathrm{C}$; $\mathrm{Rf}=0.84$ (Silica $60 \mathrm{~A}, 1: 1$ ethyl acetate: petroleum ether, $\mathrm{v} / \mathrm{v}) ; \delta_{\mathrm{H}}(1.86(2 \mathrm{H}$, $\left.\mathrm{m}, 1.6 \mathrm{~Hz}, 7.5 \mathrm{~Hz}, 2^{\mathrm{II}}\right), 2.03\left(2 \mathrm{H}, \mathrm{m}, 1.6 \mathrm{~Hz}, 7.5 \mathrm{~Hz}, 3^{\mathrm{II}}\right)$, $3.16\left(2 \mathrm{H}, \mathrm{t}, 1.6 \mathrm{~Hz}, 7.5 \mathrm{~Hz}, 2^{\mathrm{IV}}\right), 4.05(2 \mathrm{H}, \mathrm{t}, 1.6 \mathrm{~Hz}, 7.5 \mathrm{~Hz}$, $\left.4^{\mathrm{II}}\right), 4.26\left(4 \mathrm{H}, \mathrm{m}, 1.6 \mathrm{~Hz}, 7.6 \mathrm{~Hz}, 15.2 \mathrm{~Hz}, \mathbf{1}^{\mathrm{II}}, \mathbf{1}^{\mathrm{IV}}\right), 6.45(2 \mathrm{H}$, $\left.\mathrm{s}, \mathbf{3}, \mathbf{3}^{\mathbf{V I}}\right), 6.95\left(4 \mathrm{H}, \mathrm{dd}, 1.4 \mathrm{~Hz}, 8.1 \mathrm{~Hz}, \mathbf{3}^{\mathbf{I}}, \mathbf{5}^{\mathbf{I}}, \mathbf{3}^{\mathbf{V}}, \mathbf{5}^{\mathbf{V}}\right), 7.01$ $\left(1 \mathrm{H}, \mathrm{s}, 1^{\mathrm{III}}\right), 7.17\left(2 \mathrm{H}, \mathrm{dd}, 1.3 \mathrm{~Hz}, 8.1 \mathrm{~Hz}, \mathbf{8}^{\mathrm{r}} \mathbf{8}^{\mathrm{VI}}\right), 7.36(2 \mathrm{H}$, m, $\left.1.3 \mathrm{~Hz}, 8.1 \mathrm{~Hz}, 15.6 \mathrm{~Hz}, \mathbf{6}, \mathbf{6}^{\mathbf{V I}}\right), 7.55\left(4 \mathrm{H}, \mathrm{d}, 8.1 \mathrm{~Hz}, \mathbf{2}^{\mathbf{I}}\right.$, $\left.\mathbf{6}^{\mathbf{I}}, \mathbf{2}^{\mathbf{V}}, \mathbf{6}^{\mathbf{V}}\right), 7.70\left(2 \mathrm{H}, \mathrm{m}, 1.3 \mathrm{~Hz}, 8.1 \mathrm{~Hz}, 15.6 \mathrm{~Hz}, \mathbf{7}, 7^{\mathbf{V I}}\right), 7.85$ $\left(2 \mathrm{H}, \mathrm{dd}, 1.3 \mathrm{~Hz}, 8.1 \mathrm{~Hz}, \mathbf{5}, \mathbf{5}^{\mathbf{V I}}\right) ; \delta_{\mathrm{C}} 26.3\left(\mathbf{3}^{\mathbf{I I}}\right), 30.5\left(\mathbf{2}^{\mathbf{I V}}\right)$, $49.4\left(\mathbf{4}^{\mathbf{I I}}\right), 67.4\left(\mathbf{1}^{\mathbf{I V}}\right), 69.1\left(\mathbf{1}^{\mathbf{I I}}, \mathbf{2}^{\mathbf{I I}}\right), 107.3\left(\mathbf{2}, \mathbf{2}^{\mathbf{V I}}\right), 115.4\left(\mathbf{3}^{\mathbf{I}}\right.$, $\left.\mathbf{5}^{\mathbf{I}}, \mathbf{3}^{\mathbf{V}}, \mathbf{5}^{\mathbf{V}}\right), 118.1\left(\mathbf{8}, \mathbf{8}^{\mathbf{V I}}\right), 122.4\left(\mathbf{1}^{\mathbf{I}}, \mathbf{1}^{\mathbf{V}}\right), 123.4\left(\mathbf{6}, \mathbf{6}^{\mathbf{V I}}\right), 124.4$ $\left(\mathbf{1}^{\mathbf{I I I}}\right), 125.7\left(\mathbf{5}, \mathbf{5}^{\mathbf{V I}}\right), 128.5\left(\mathbf{2}^{\mathrm{I}}, \mathbf{6}^{\mathbf{I}}, \mathbf{2}^{\mathbf{V}}, \mathbf{6}^{\mathrm{V}}\right), 131.2\left(\mathbf{7}, \mathbf{7}^{\mathbf{V I}}\right)$, $142.9\left(\mathbf{2}^{\mathrm{III}}\right), 155.7\left(\mathbf{9}, \mathbf{9}^{\mathrm{VI}}\right), 158.5\left(\mathbf{4}^{\mathbf{V}}\right), 160.5\left(\mathbf{4}^{\prime}\right), 163.6(\mathbf{1}$, $\left.\mathbf{1}^{\mathrm{VI}}\right), 177.2\left(\mathbf{4}, \mathbf{4}^{\mathrm{VI}}\right)$; LCMS ESI $\left(\mathrm{MeOH}, 2500 \mathrm{~V}, 300^{\circ} \mathrm{C}\right)=$ $\mathrm{m} / \mathrm{z}[\mathrm{M}+\mathrm{H}]^{+}=626.23$; FTIR (ATR diamond $4 \mathrm{~cm}^{-1}$ ): 1160 $\mathrm{cm}^{-1}$ (C-O-C, large ring C-O stretch), 1051 and $1238 \mathrm{~cm}^{-1}$ (C-O, phenolic), $1595 \mathrm{~cm}^{-1} \quad\left(\mathrm{C}=\mathrm{C}\right.$ aromatic), $1661 \mathrm{~cm}^{-1}$ (C=O, ketone), $1451 \mathrm{~cm}^{-1}$ and $1732 \mathrm{~cm}^{-1}$ (triazole ring), 2980 $\mathrm{cm}^{-1}$ (C-H, alkane), $3301 \mathrm{~cm}^{-1}$ (C-H, aromatic).

\section{2-(4'-(3-(4-(3-(4-(4-Oxo-4H-chromen-2- yl)phenoxy)propyl)-1H-1,2,3-triazol-1- yl)propoxy)phenyl)-4H-chromen-4-one - $\underline{\mathbf{1 7}}$.}

Yield: 0.282 g, $74.2 \%$; m.p. $274-276{ }^{\circ} \mathrm{C} \mathrm{Rf}=0.85$ (Silica $60 \mathrm{~A}, 1: 1$ ethyl acetate: petroleum ether, $\mathrm{v} / \mathrm{v}) ; \delta_{\mathrm{H}}(2.21(4 \mathrm{H}$, m, $\left.1.6 \mathrm{~Hz}, 3.8 \mathrm{~Hz}, 7.4 \mathrm{~Hz}, 14.8 \mathrm{~Hz}, \mathbf{2}^{\mathrm{II}}, \mathbf{2}^{\mathrm{IV}}\right), 2.65(2 \mathrm{H}, \mathrm{t}, 1.6$ $\left.\mathrm{Hz}, 7.4 \mathrm{~Hz}, 3^{\mathbf{I V}}\right), 4.06\left(2 \mathrm{H}, \mathrm{t}, 1.6 \mathrm{~Hz}, 7.4 \mathrm{~Hz}, \mathbf{3}^{\mathbf{I I}}\right), 4.26(4 \mathrm{H}$, m, $\left.1.6 \mathrm{~Hz}, 7.4 \mathrm{~Hz}, 14.8 \mathrm{~Hz}, \mathbf{1}^{\mathbf{I I}}, \mathbf{1}^{\mathbf{I V}}\right), 6.45\left(2 \mathrm{H}, \mathrm{s}, \mathbf{3}, \mathbf{3}^{\mathbf{V I}}\right)$, $6.95\left(4 \mathrm{H}, \mathrm{dd}, 1.4 \mathrm{~Hz}, 8.1 \mathrm{~Hz}, \mathbf{3}^{\mathbf{I}}, \mathbf{5}^{\mathbf{I}}, \mathbf{3}^{\mathbf{V}}, \mathbf{5}^{\mathbf{V}}\right), 7.01\left(1 \mathrm{H}, \mathrm{s}, 1^{\mathrm{III}}\right)$, $7.17\left(2 \mathrm{H}, \mathrm{dd}, 1.3 \mathrm{~Hz}, 8.1 \mathrm{~Hz}, \mathbf{8}, \mathbf{8}^{\mathbf{V I}}\right), 7.36(2 \mathrm{H}, \mathrm{m}, 1.3 \mathrm{~Hz}$, $\left.8.1 \mathrm{~Hz}, 15.6 \mathrm{~Hz}, \mathbf{6}, \mathbf{6}^{\mathbf{V I}}\right), 7.55\left(4 \mathrm{H}, \mathrm{d}, 8.1 \mathrm{~Hz}, \mathbf{2}^{\mathbf{I}}, \mathbf{6}^{\mathbf{I}}, \mathbf{2}^{\mathbf{V}}, \mathbf{6}^{\mathbf{V}}\right)$, $7.70\left(2 \mathrm{H}, \mathrm{m}, 1.3 \mathrm{~Hz}, 8.1 \mathrm{~Hz}, 15.6 \mathrm{~Hz}, 7,7^{\mathrm{VI}}\right), 7.85(2 \mathrm{H}, \mathrm{dd}$, $\left.1.3 \mathrm{~Hz}, 8.1 \mathrm{~Hz}, \mathbf{5}, \mathbf{5}^{\mathbf{V I}}\right) ; \delta_{\mathrm{C}}\left(26.3\left(\mathbf{3}^{\mathrm{II}}\right), 25.5\left(\mathbf{3}^{\mathbf{I V}}\right), 28.9\left(\mathbf{2}^{\mathbf{I V}}\right)\right.$, $49.4\left(\mathbf{3}^{\text {II }}\right), 62.6\left(\mathbf{2}^{\mathbf{I I}}\right), 69.1\left(\mathbf{1}^{\mathbf{I V}}\right), 70.8\left(\mathbf{1}^{\mathrm{II}}\right), 107.3\left(\mathbf{2}, \mathbf{2}^{\mathrm{VI}}\right)$, $115.4\left(\mathbf{3}^{\mathbf{I}}, \mathbf{5}^{\mathbf{I}}, \mathbf{3}^{\mathbf{V}}, \mathbf{5}^{\mathbf{V}}\right), 118.1\left(\mathbf{8}, \mathbf{8}^{\mathbf{V I}}\right), 122.4\left(\mathbf{1}^{\mathbf{I}}, \mathbf{1}^{\mathbf{V}}\right), 123.4(\mathbf{6}$, $\left.\mathbf{6}^{\mathrm{VI}}\right), 124.4\left(\mathbf{1}^{\mathrm{III}}\right), 125.7\left(\mathbf{5}, \mathbf{5}^{\mathrm{VI}}\right), 128.5\left(\mathbf{2}^{\mathbf{I}}, \mathbf{6}^{\mathbf{I}}, \mathbf{2}^{\mathbf{V}}, \mathbf{6}^{\mathbf{V}}\right), 131.2$ $\left(\mathbf{7}, 7^{\mathrm{VI}}\right), 142.9\left(\mathbf{2}^{\mathrm{II}}\right), 155.7\left(\mathbf{9}^{\mathrm{II}} \mathbf{9}^{\mathrm{VI}}\right), 158.5\left(\mathbf{4}^{\mathrm{V}}\right), 160.5\left(\mathbf{4}^{\prime}\right)$, $163.6\left(\mathbf{1}, \mathbf{1}^{\mathrm{VI}}\right), 177.2\left(\mathbf{4}, \mathbf{4}^{\mathrm{VI}}\right)$; LCMS ESI $(\mathrm{MeOH}, 2500 \mathrm{~V}$, $300^{\circ} \mathrm{C}$ ) $=\mathrm{m} / \mathrm{z}[\mathrm{M}+\mathrm{H}]^{+}=626.22 ; \quad$ FTIR (ATR diamond $\left.4 \mathrm{~cm}^{-1}\right): 1165 \mathrm{~cm}^{-1}$ (C-O-C, large ring C-O stretch), 1053 and 
$1241 \mathrm{~cm}^{-1}$ (C-O, phenolic), $1597 \mathrm{~cm}^{-1} \quad(\mathrm{C}=\mathrm{C}$ aromatic), $1663 \mathrm{~cm}^{-1}$ (C=O, ketone), $1452 \mathrm{~cm}^{-1}$ and $1731 \mathrm{~cm}^{-1}$ (triazole ring), $2983 \mathrm{~cm}^{-1}$ (C-H, alkane), $3304 \mathrm{~cm}^{-1}$ (C-H, aromatic).

\section{2-(4'-(3-(1-(4-(4-(4-Oxo-4H-chromen-2-}

\section{yl)phenoxyl)butyl)-1H-1,2,3-triazol-4-}

\section{yl)propoxy)phenyl)-4H-chromen-4-one - 18 .}

Yield: 0.301 g, $75.2 \%$; m.p. $282-283{ }^{\circ} \mathrm{C} ; \mathrm{Rf}=0.87$ (Silica $60 \mathrm{~A}, 1: 1$ ethyl acetate: petroleum ether, $\mathrm{v} / \mathrm{v}) ; \delta_{\mathrm{H}} 1.86(2 \mathrm{H}$, m, $\left.1.5 \mathrm{~Hz}, 7.5 \mathrm{~Hz}, 15.0 \mathrm{~Hz}, 2^{\mathrm{II}}\right), 2.02(2 \mathrm{H}, \mathrm{m} 1.5 \mathrm{~Hz} 7.5 \mathrm{~Hz}$, $\left.15.0 \mathrm{~Hz}, \mathbf{3}^{\text {II }}\right) 2.21\left(2 \mathrm{H}, \mathrm{m}, 1.5 \mathrm{~Hz}, 7.5 \mathrm{~Hz}, 15.0 \mathrm{~Hz}, \mathbf{2}^{\text {IV }}\right), 2.65$ $\left(2 \mathrm{H}, \mathrm{t}, 1.5 \mathrm{~Hz}, 7.5 \mathrm{~Hz}, 3^{\mathrm{IV}}\right), 4.04\left(2 \mathrm{H}, \mathrm{t}, 1.5 \mathrm{~Hz}, 7.5 \mathrm{~Hz}, 4^{\mathrm{II}}\right)$, $4.25\left(4 \mathrm{H}, \mathrm{m}, 1.5 \mathrm{~Hz}, 7.5 \mathrm{~Hz}, 15.0 \mathrm{~Hz}, \mathbf{1}^{\mathbf{I I}}, \mathbf{1}^{\mathbf{I V}}\right), 6.45(2 \mathrm{H}, \mathrm{s}$, $\left.\mathbf{3}, \mathbf{3}^{\mathbf{V I}}\right), 6.95\left(4 \mathrm{H}, \mathrm{dd}, 1.4 \mathrm{~Hz}, 8.1 \mathrm{~Hz}, \mathbf{3}^{\mathbf{I}}, \mathbf{5}^{\mathbf{I}}, \mathbf{3}^{\mathbf{V}}, \mathbf{5}^{\mathbf{V}}\right), 7.01(1 \mathrm{H}$, s, $\left.1^{\mathrm{III}}\right), 7.17\left(2 \mathrm{H}, \mathrm{dd}, 1.3 \mathrm{~Hz}, 8.1 \mathrm{~Hz}, \mathbf{8}, \mathbf{8}^{\mathrm{VI}}\right), 7.36(2 \mathrm{H}, \mathrm{m}, 1.3$ $\left.\mathrm{Hz}, 8.1 \mathrm{~Hz}, 15.6 \mathrm{~Hz}, \mathbf{6}, \mathbf{6}^{\mathbf{V I}}\right), 7.55\left(4 \mathrm{H}, \mathrm{d}, 8.1 \mathrm{~Hz}, \mathbf{2}^{\mathbf{I}}, \mathbf{6}^{\mathbf{I}}, \mathbf{2}^{\mathbf{V}}\right.$, $\left.6^{\mathbf{V}}\right), 7.70\left(2 \mathrm{H}, \mathrm{m}, 1.3 \mathrm{~Hz}, 8.1 \mathrm{~Hz}, 15.6 \mathrm{~Hz}, \mathbf{7}^{-} 7^{\mathrm{VI}}\right), 7.85(2 \mathrm{H}$, dd, $\left.1.3 \mathrm{~Hz}, 8.1 \mathrm{~Hz}, \mathbf{5}, \mathbf{5}^{\mathbf{V I}}\right) ; \delta_{\mathrm{C}}\left(25.5\left(3^{\mathbf{I V}}\right), 26.3\left(\mathbf{3}^{\mathbf{I I}}\right), 28.9\right.$ $\left(\mathbf{2}^{\mathbf{I I}}\right), 49.4\left(\mathbf{4}^{\mathbf{I I}}\right), 69.1\left(\mathbf{1}^{\mathbf{I I}}, \mathbf{2}^{\mathrm{II}}, \mathbf{1}^{\mathbf{I V}}\right), 107.3\left(\mathbf{2}, \mathbf{2}^{\mathbf{V I}}\right), 115.4\left(\mathbf{3}^{\mathbf{I}}\right.$, $\left.\mathbf{5}^{\mathbf{I}}, \mathbf{3}^{\mathbf{V}}, \mathbf{5}^{\mathbf{V}}\right), 118.1\left(\mathbf{8}, \mathbf{8}^{\mathbf{V I}}\right), 122.4\left(\mathbf{1}^{\mathbf{I}}, \mathbf{1}^{\mathbf{V}}\right), 123.4\left(\mathbf{6}, \mathbf{6}^{\mathbf{V I}}\right), 124.4$ $\left(\mathbf{1}^{\mathrm{III}}\right), 125.7\left(\mathbf{5}, \mathbf{5}^{\mathbf{V I}}\right), 128.5\left(\mathbf{2}^{\mathrm{I}}, \mathbf{6}^{\mathbf{I}}, \mathbf{2}^{\mathbf{V}}, \mathbf{6}^{\mathrm{V}}\right), 131.2\left(\mathbf{7}, \mathbf{7}^{\mathbf{V I}}\right)$, $142.9\left(\mathbf{2}^{\mathrm{III}}\right), 155.7\left(\mathbf{9}, \mathbf{9}^{\mathrm{VI}}\right), 158.5\left(\mathbf{4}^{\mathrm{V}}\right), 160.5\left(\mathbf{4}^{\prime}\right), 163.6(\mathbf{1}$, $\left.\mathbf{1}^{\mathrm{VI}}\right), 177.2\left(\mathbf{4}, \mathbf{4}^{\mathrm{VI}}\right)$; LCMS ESI $\left(\mathrm{MeOH}, 2500 \mathrm{~V}, 300^{\circ} \mathrm{C}\right)=$ $\mathrm{m} / \mathrm{z}[\mathrm{M}+\mathrm{H}]^{+}=640.25$; FTIR (ATR diamond $4 \mathrm{~cm}^{-1}$ ): 1160 $\mathrm{cm}^{-1}$ (C-O-C, large ring C-O stretch), 1051 and $1238 \mathrm{~cm}^{-1}$ (C-O, phenolic), $1595 \mathrm{~cm}^{-1} \quad\left(\mathrm{C}=\mathrm{C}\right.$ aromatic), $1661 \mathrm{~cm}^{-1}$ (C=O, ketone), $1451 \mathrm{~cm}^{-1}$ and $1732 \mathrm{~cm}^{-1}$ (triazole ring), 2980 $\mathrm{cm}^{-1}$ (C-H, alkane), $3301 \mathrm{~cm}^{-1}$ (C-H, aromatic).

\subsection{Cell culture and growth inhibition studies}

RPMI-1640 supplemented with $10 \%$ heat inactivated FBS, L-glutamine $(1.0 \mathrm{mM})$ and streptomycin $(1.0 \mathrm{mM})$ was used to culture Hep G2, MCF7, HCT116 and MOLT-4 cell lines. McCoys 5A supplemented with $10 \%$ heat inactivated FBS, L-glutamine $(1.0 \mathrm{mM})$ and streptomycin $(1.0 \mathrm{mM})$ was used to culture the $\mathrm{HaCaT}$ cell line. Culture was undertaken in a humidified incubator with $5 \% \mathrm{CO}_{2}$ at $37^{\circ} \mathrm{C}$ until $75 \%$ confluence was reached. The general method undertaken for growth inhibition studies used cells grown in T-75 cell culture flasks before being aliquoted into sterile 96-well plates and incubated for 12 hours. The cells were then treated with a concentration gradient $(125-1.9 \mu \mathrm{M})$ of bis-flavone dimer for 48 hours. Following this the cells were aspirated and $\quad 30.0 \mu \mathrm{L}$ of 3-(4,5-dimethylthiazol-2-yl)-2,5diphenyltetrazolium bromide (MTT) $(5.0 \mathrm{mg} / \mathrm{ml}$ in PBS) were added. The plate was incubated for 3.5 hours at $37^{\circ} \mathrm{C}$ in an environment of $5 \% \mathrm{CO}_{2}$ before the wells were aspirated and $100 \mu \mathrm{L}$ of DMSO added. The 96 well plates were agitated using an orbital stirrer for 60 seconds before absorbance readings at $570 \mathrm{~nm}$ and $630 \mathrm{~nm}$ were taken using a Thermo Labsystems MultiSkan Ascent system. For suspension cell lines the same method was undertaken however MTS was used in place of MTT, following manufacturer's guidelines (Promega, UK). The growth inhibition studies using MTT/MTS assays were performed on a Thermo Labsystems MultiSkan Ascent system.

\section{CONCLUSION}

In summary, we have shown that through the linking of two mono-flavones it is possible to form a bis-flavone dimer. The synthetic procedures developed to produce both the mono- flavones 6-7 and bis-flavone dimers 8-18 were designed and optimized to produce high yields of the novel compounds utilising green chemistry conditions. The absence of harsh conditions, prolonged heating and the generally accessible required reagents will allow for the synthesis of dimers of additional analogues in this series to further evaluate biological activity. This study has highlighted the advantages of combining two mono-flavones with an efficient and green synthetic approach is successful for the production of new molecules. Bis-flavone dimer $\mathbf{1 6}$ was identified as the lead molecule from this study as it caused substantial growth inhibition in a number of cell lines. The carbon chains which link the two mono-flavone moieties, are two and three carbons in length with a central 1,2,3-triazole ring. Dimeric approaches to produce anticancer agents has been explored and produced interesting results. Dimers based on quinones, coumarins, and acridines have shown a greater interaction with heterodimer and homodimer proteins as well with DNA[28]. This principle was followed in this new family of flavonoids. This study has highlighted the advantages of combining two mono-flavones in order to produce more selective and highly active bis-flavone dimers. The identification of the lead bis-flavone dimer, 16, and the development of efficient and green synthetic methods will allow the extension of this study to further elucidate the parameters that control activity and selectivity in this novel class of agent.

\section{CONFLICT OF INTEREST}

We would like to thanks Salford Advantage Fund and Salford Prime fund for collaborating with consumables in this project. Authors declare there is no conflict of interest.

\section{ACKNOWLEDGEMENTS}

We would like to thank the technical staff at the University of Salford and Salford Analytical Services in particular $\mathrm{Mr}$ Kirit Amin for his outstanding analytical contributions. We would also like to express our gratitude to Dr Nanda Puspita, Dr Patrick Killoran and Dr Firozeh Ashnti for advice.

\section{SUPPLEMENTARY MATERIAL}

Added as separate file

\section{REFERENCES}

(1) Fusi, F.; Spiga, O.; Trezza, A.; Sgaragli, G.; Saponara, S. Eur J Pharmac 2017, 796,158-174.

(2) Lin, Y.; Shi, R.; Wang, X.; Shen, H.M. Curr. Cancer Drug Targets 2008, 8,634-646.

(3) Middleton, E. Jr.; Kandaswami, C.; Theoharides, T.C. Pharmacological Rev. 2000, 52,673-751.

(4) Ishaq, M.S.; Afridi, M.S.; Khattak, M.; Ahmad, S. Sci. World J. 2014, 2014:ID 269793. http://dx.doi.org/10.1155/2014/269793

(5) Mujeeb, F.; Bajpai, P.; Pathak, N. Biomed Res. Int. 2014, 2014:ID 497606. http://dx.doi.org/10.1155/2014/497606

(6) Lin, B.W.; Gong, C.C.; Song, H.F. Br. J. Pharmacol. 2016, 174,1226-1243.

(7) Ragazzon, P.A.; Bradshaw, T.; Matthews, C.; Iley, J.; Missailidis, S. Anticancer Res. 2009, 29, 2273-2283. 
(8) Tanemossua, S.A.; Franke, K.; Schmidt, A.N.; Wabo, H.K.; Tane, P.; Wessjohanna, L. Phytochemistry 2015, 105, 171-177.

(9) Nakashima, K.; Abe, N.; Kamiya, K.; Ito, T.; Oyama, M.; Iinuma, M. Helv. Chim. Acta 2009, 92, 1999-2008.

(10) Ramaswamy, A.S.; Basu, N. Vasopeptides 1972, 46, 357-360.

(11) Pelter, A. Tetrahederon Lett. 1967, 19, 1767-1771.

(12) Roitman, J.N.; Wong, R.Y.; Wollenweber, E. Phytochemistry 1993, 34,297-301.

(13) Sawada, T. YAKUGAKU ZASSHI. 1958, 78, 10231027.

(14) Mahal, H.S.; Venkataraman, K. J. Chem. Soc. 1934, 56,1767-1769.

(15) Ares, J.J.; Outt, P.E.; Kakodkar, S.V.; Buss, R.C.; Geiger, J.C. J. Org. Chem. 1993, 58, 7903-7905.

(16) Kshatriya, R.B.; Shaikh, Y.I.; Nazeruddin, G.M. Orient. J. Chem. 2013, 29, 1475-1487.

(17) Bálint, E.; Kovács, O.; Drahos, L.; Keglevich, G. Lett. Org. Chem. 2013, 10, 330-336.

(18) Lepore, S.D.; He, T. J. Org. Chem. 2003, 68, 82618263.

(19) Ragazzon, P.A.; Iley, J.; Missailidis, S.. Anticancer Res. 2009, 29, 2285-2294.

(20) Kolb, H.C.; Finn, M.G.; Sharpless, K.B. Angew. Chem. Int. Ed. 2001, 40, 2004-2021.

(21) Woo, H.; Kang, H.; Kim, A.; Jang, S.; Park, J.C.; Park, S.; Kim, B.S.; Song, H.; Park, K.H. Molecules 2012, 17, 13235-13252.

(22) Zhang, F.; Moses, J.E. Org. Lett. 2009, 11, 1587-1590.

(23) Rostovtsev, V.V.; Green, L.G.; Fokin, V.V.; Sharpless, K.B. Angew. Chem. Int. Ed. 2002, 41, 2596-2599.

(24) McKeage, M.J.; Baguley, B.C. Cancer 2010, 116, 1859-1871.

(25) Merlo, D.F.; Filiberti, R.; Kobernus, M.; Bartonova, A.; Gamulin, M.; Ferencic, Z.; Dusinska, M.; Fucic, A., Environ. Health 2012, 11:S9 doi: 10.1186/1476069X-11-S1-S9.

(26) Hochberg, M.E.; Noble, R.J. Ecol. Lett.; 2017, 20,117-134.

(27) Hadden, M.K.; Blagg, B.S.J. Anticancer Agents Med. Chem. 2008, 8, 807-816. 